\title{
Common fixed point results for probabilistic $\varphi$-contractions in generalized probabilistic metric spaces
}

\author{
Jingfeng Tian ${ }^{\mathrm{a}, *}$, Ximei $\mathrm{Hu}^{\mathrm{b}}$ \\ ${ }^{a}$ College of Science and Technology, North China Electric Power University, Baoding, Hebei Province, 071051, P. R. China. \\ ${ }^{b}$ China Mobile Group Hebei Co., Ltd., Baoding, Hebei Province, 071000, P. R. China.
}

Communicated by B. Samet

\begin{abstract}
In this paper, we present some new fixed point and common fixed point (common coupled fixed point, common tripled fixed point, and common quadruple fixed point) theorems of probabilistic contractions with a gauge function $\varphi$ in generalized probabilistic metric spaces proposed by Zhou et al. [C.-L. Zhou, S.-H. Wang, L. Ćirić, S. M. Alsulami, Fixed Point Theory Appl., 2014 (2014), 15 pages]. Our results extend some existing results. Moreover, an example is given to illustrate our main results. (C) 2017 All rights reserved.
\end{abstract}

Keywords: Coupled fixed point, fixed point, metric space, probabilistic $\varphi$-contractions, gauge function. 2010 MSC: 47H10, 54E70.

\section{Introduction}

The notion of a coupled fixed point was introduced by Opoĭcev [17], and then Guo and Lakshmikantham [6] in 1987 proved some fixed point theorems for coupled fixed point under certain conditions in Banach space. Later, Bhaskar and Lakshmikantham [5] proved the existence and uniqueness of a coupled fixed point result under a weak contractivity condition in the context of partially ordered metric spaces. In 2009, Lakshmikantham and Ćirić [13] extended the result by introducing the notion of the g-monotone property. Based on Lakshmikantham and Ćirić's work, many researchers have obtained more coupled fixed point theorems in metric space; see [4, 10,11, 14, 18]. Recently, the investigation of coupled fixed point theorem has been extended from metric spaces to Menger probabilistic metric spaces. For example, using the properties of the pseudo-metric and the triangular norm, Xiao et al. [22] gave some common coupled fixed point theorems in Menger probabilistic metric spaces. Ćirić et al. [3] established some coupled fixed point theorems for mixed monotone mappings in the partially ordered Menger probabilistic metric spaces. In 2014, $\mathrm{Wu}$ [21] presented some coupled fixed point theorems for nonlinear contractive operators in partially ordered Menger probabilistic metric spaces, which improved and generalized some main results of Ćirić et al. [3].

\footnotetext{
*Corresponding author

Email addresses: tianjf@ncepu.edu.cn (Jingfeng Tian), huxm_bd@163.com (Ximei Hu)
} 
The concept of tripled fixed point was introduced by Berinde and Borcut [2]. In their manuscript, some new tripled point theorems are obtained. In 2012, Karapinar and Luong [12] presented the notion of quadruple fixed point and proved the related fixed point theorems.

Inspired by the works of [2], [12], and [22], in this paper, we try to give some new fixed point and common fixed point (common coupled fixed point, common tripled fixed point, and common quadruple fixed point) theorems under probabilistic $\varphi$-contractive in generalized probabilistic metric spaces proposed by Zhou et al. [23]. The obtained results generalize the corresponding ones from [22].

\section{Preliminaries}

Suppose that $\mathbb{R}=(-\infty,+\infty), \mathbb{R}^{+}=[0,+\infty), \overline{\mathbb{R}}=\mathbb{R} \cup\{-\infty,+\infty\}$, and let $\mathbb{Z}^{+}$be the set of all positive integers. A function $G: \overline{\mathbb{R}} \rightarrow[0,1]$ is called a distribution function if it is nondecreasing and leftcontinuous with $F(-\infty)=0, F(+\infty)=1$. The set of all probability distribution functions is denoted by $\mathcal{D}_{\infty}$. Suppose that $\mathcal{D}=\left\{F \in \mathcal{D}_{\infty}: \inf _{\mathrm{t} \in \mathbb{R}} F(t)=0\right.$, $\left.\sup _{t \in \mathbb{R}} F(t)=1\right\}, \mathcal{D}_{\infty}^{+}=\left\{F \in \mathcal{D}_{\infty}: F(0)=0\right\}$, and $\mathcal{D}^{+}=\mathcal{D} \cap \mathcal{D}_{\infty}^{+}$.

Definition 2.1 ([19]). A mapping $\Delta:[0,1] \times[0,1] \rightarrow[0,1]$ is a continuous t-norm if $\Delta$ satisfies the following conditions:

(1) $\Delta$ is commutative and associative, i.e., $\Delta(a, b)=\Delta(b, a)$ and $\Delta(a, \Delta(b, c))=\Delta(\Delta(a, b), c)$ for all $a, b, c \in[0,1]$;

(2) $\Delta$ is continuous;

(3) $\Delta(a, 1)=a$ for all $a \in[0,1]$;

(4) $\Delta(\mathrm{a}, \mathrm{b}) \leqslant \Delta(\mathrm{c}, \mathrm{d})$ whenever $\mathrm{a} \leqslant \mathrm{c}$ and $\mathrm{b} \leqslant \mathrm{d}$ for all $\mathrm{a}, \mathrm{b}, \mathrm{c}, \mathrm{d} \in[0,1]$.

From the definition of $\Delta$, it follows that $\Delta(a, b) \leqslant \min \{a, b\}$ for all $a, b \in[0,1]$.

Two typical examples of continuous t-norm are $\Delta_{M}(a, b)=\min \{a, b\}$ and $\Delta_{p}(a, b)=a b$ for all $a, b \in[0,1]$.

Definition 2.2 ([7]). A t-norm $\Delta$ is said to be of H-type (Hadžić type) if a family of functions $\left\{\Delta^{\mathrm{n}}(\mathrm{t})\right\}_{\mathfrak{n}=1}^{+\infty}$ is equicontinuous at $t=1$, that is, for any $\epsilon \in(0,1)$, there exists $\delta \in(0,1)$ such that

$$
\mathrm{t}>1-\delta \Rightarrow \Delta^{\mathrm{n}}(\mathrm{t})>1-\epsilon
$$

for all $n \geqslant 1$, where $\Delta^{n}:[0,1] \rightarrow[0,1]$ is defined as follows:

$$
\Delta^{1}(\mathrm{t})=\Delta(\mathrm{t}, \mathrm{t}), \Delta^{2}(\mathrm{t})=\Delta\left(\mathrm{t}, \Delta^{1}(\mathrm{t})\right), \cdots, \Delta^{\mathrm{n}}(\mathrm{t})=\Delta\left(\mathrm{t}, \Delta^{\mathrm{n}-1}(\mathrm{t})\right), \cdots
$$

Obviously, $\Delta^{\mathrm{n}}(\mathrm{t}) \leqslant \mathrm{t}$ for any $\mathrm{n} \in \mathrm{N}$ and $\mathrm{t} \in[0,1]$.

$\Delta_{M}$ is a trivial example of t-norm of Hadzić-type [8].

Definition 2.3. If $\varphi: \mathbb{R}^{+} \rightarrow \mathbb{R}^{+}$is a function such that $\varphi(0)=0$, then $\varphi$ is called a gauge function. If $\mathrm{t} \in \mathbb{R}^{+}$, then $\varphi^{\mathrm{n}}(\mathrm{t})$ denotes the $\mathrm{nth}$ iteration of $\varphi(\mathrm{t})$ and $\varphi^{-1}(\{0\})=\left\{\mathrm{t} \in \mathbb{R}^{+}: \varphi(\mathrm{t})=0\right\}$.

Definition 2.4 ([15]). A Menger probabilistic metric space (shortly, Menger PM-space) is a triple $(X, \mathcal{F}, \Delta)$, where $X$ is a nonempty set, $\Delta$ is a continuous t-norm, and $\mathcal{F}$ is a mapping from $X \times X \rightarrow \mathcal{D}_{\infty}^{+}\left(F_{x, y}\right.$ denotes the value of $\mathcal{F}$ at the pair $(x, y))$ satisfying the following conditions:

(PM-1) $F_{x, y}(t)=1$ for all $x, y \in X$ and $t>0$ if and only if $x=y$;

(PM-2) $F_{x, y}(t)=F_{y, x}(t)$ for all $x, y \in X$ and $t>0$;

$(P M-3) F_{x, z}(t+s) \geqslant \Delta\left(F_{x, y}(t), F_{y, z}(s)\right)$ for all $x, y, z \in X$ and every $s>0, t>0$.

Definition 2.5 ([16]). Let $X$ be a nonempty set and $G: X \times X \times X: \rightarrow \mathbb{R}^{+}$be a function satisfying the following properties: 
(G1) $G(x, y, z)=0$ if $x=y=z$,

(G2) $0<G(x, x, y)$ for all $x, y \in X$ with $x \neq y$,

(G3) $G(x, x, y) \leqslant G(x, y, z)$ for all $x, y, z \in X$ with $y \neq z$,

(G4) $G(x, y, z)=G(x, z, y)=G(y, z, x)=\cdots$ for all $x, y, z \in X$ (symmetry in all three variables),

(G5) $G(x, y, z) \leqslant G(x, a, a)+G(a, y, z)$ for all $x, y, z, a \in X$ (rectangle inequality).

Then the function $G$ is called a generalized metric or, more specifically, a G-metric on $X$, and the pair $(\mathrm{X}, \mathrm{G})$ is called a $\mathrm{G}$-metric space.

In 2014, Zhou et al. [23] introduced the following probabilistic version of G-metric space, which is a generalization of Menger PM-space.

Definition 2.6 ([23]). A Menger probabilistic G-metric space (briefly, PGM-space) is a triple $\left(X, G^{*}, \Delta\right)$, where $X$ is a nonempty set, $\Delta$ is a continuous t-norm, and $G^{*}$ is a mapping from $X \times X \times X$ into $\mathcal{D}_{\infty}^{+}\left(G_{x, y, z}^{*}\right.$ denotes the value of $G^{*}$ at the point $\left.(x, y, z)\right)$ satisfying the following conditions:

(PGM-1) $G_{x, y, z}^{*}(t)=1$ for all $x, y, z \in X$ and $t>0$ if and only if $x=y=z$;

(PGM-2) $\mathrm{G}_{x, x, y}^{*}(t) \geqslant \mathrm{G}_{x, y, z}^{*}(t)$ for all $x, y \in X$ with $z \neq y$ and $t>0$;

(PGM-3) $G_{x, y, z}^{*}(t)=G_{x, z, y}^{*}(t)=G_{y, x, z}^{*}(t)=\cdots$ (symmetry in all three variables);

$(P G M-4) G_{x, y, z}^{*}(t+s) \geqslant \Delta\left(G_{x, a, a}^{*}(s), G_{a, y, z}^{*}(t)\right)$ for all $x, y, z, a \in X$ and every $s>0, t>0$.

Definition 2.7 ([23]). Let $\left(X, G^{*}, \Delta\right)$ be a PGM-space and $x_{0}$ be any point in $X$. For any $\epsilon>0$ and $\delta$ with $0<\delta<1$, an $(\epsilon, \delta)$-neighborhood of $x_{0}$ is the set of all points $y$ in $X$ for which $G_{x_{0, y}, y}^{*}(\epsilon)>1-\delta$ and $\mathrm{G}_{y, x_{0}, x_{0}}^{*}(\epsilon)>1-\delta$. We write

$$
\mathrm{N}_{x_{0}}(\epsilon, \delta)=\left\{y \in X: G_{x_{0, y}, y}^{*}(\epsilon)>1-\delta, G_{y, x_{0}, x_{0}}^{*}(\epsilon)>1-\delta\right\}
$$

Definition 2.8 ([23]).

(1) A sequence $\left\{x_{n}\right\}$ in a PGM-space $\left(X, G^{*}, \Delta\right)$ is said to be convergent to a point $x \in X$ (write $x_{n} \rightarrow x$ ) if, for any $\epsilon>0$ and $0<\delta<1$, there exists a positive integer $M_{\epsilon, \delta}$ such that $x_{n} \in N_{x}(\epsilon, \delta)$ whenever $n>M_{\epsilon, \delta}$.

(2) A sequence $\left\{x_{n}\right\}$ in a PGM-space $\left(X, G^{*}, \Delta\right)$ is called a Cauchy sequence if, for any $\epsilon>0$ and $0<\delta<1$, there exists a positive integer $M_{\epsilon, \delta}$ such that $G_{x_{n}, x_{m}, x_{l}}^{*}(\epsilon)>1-\delta$ whenever $m, n, l>M_{\epsilon, \delta}$.

(3) A PGM-space $\left(X, G^{*}, \Delta\right)$ is said to be complete if every Cauchy sequence in $X$ converges to a point in $X$.

Theorem 2.9 ([23]). Let $\left(X, G^{*}, \Delta\right)$ be a PGM-space. Let $\left\{x_{n}\right\},\left\{y_{n}\right\}$, and $\left\{z_{n}\right\}$ be sequences in $X$ and $x, y, z \in X$. If $\mathrm{x}_{\mathrm{n}} \rightarrow \mathrm{x}, \mathrm{y}_{\mathrm{n}} \rightarrow \mathrm{y}$, and $z_{\mathrm{n}} \rightarrow z$ as $\mathrm{n} \rightarrow \infty$, then, for any $\mathrm{t}>0, \mathrm{G}_{\mathrm{x}_{\mathrm{n}}, \mathrm{y}_{\mathrm{n}}, z_{\mathrm{n}}}^{*}(\mathrm{t}) \rightarrow \mathrm{G}_{\mathrm{x}, \mathrm{y}, \mathrm{z}}^{*}(\mathrm{t})$ as $\mathrm{n} \rightarrow \infty$.

Lemma 2.10 ([9]). Suppose that $\mathrm{F} \in \mathcal{D}^{+}$. For each $\mathrm{n} \in \mathbb{Z}^{+}$, let $\mathrm{F}_{\mathrm{n}}: \mathbb{R} \rightarrow[0,1]$ be nondecreasing, and $\mathrm{g}_{\mathrm{n}}$ : $(0,+\infty) \rightarrow(0,+\infty)$ satisfy $\lim _{n \rightarrow \infty} g_{n}(t)=0$ for any $t>0$. If

$$
F_{n}\left(g_{n}(t)\right) \geqslant F(t)
$$

for any $\mathrm{t}>0$, then $\lim _{\mathrm{n} \rightarrow \infty} \mathrm{F}_{\mathrm{n}}(\mathrm{t})=1$ for any $\mathrm{t}>0$.

Definition 2.11. A point $\left(x_{1}, \ldots, x_{n}\right) \in X^{n}(n=1,2,3,4)$ is

(1) a coupled fixed point [5] if $n=2, T\left(x_{1}, x_{2}\right)=x_{1}$ and $T\left(x_{2}, x_{1}\right)=x_{2}$;

(2) a tripled fixed point [2] if $n=3, T\left(x_{1}, x_{2}, x_{3}\right)=x_{1}, T\left(x_{2}, x_{1}, x_{3}\right)=x_{2}$, and $T\left(x_{3}, x_{1}, x_{2}\right)=x_{3}$;

(3) a quadruple fixed point [12] if $n=4, T\left(x_{1}, x_{2}, x_{3}, x_{4}\right)=x_{1}, T\left(x_{2}, x_{1}, x_{3}, x_{4}\right)=x_{2}, T\left(x_{3}, x_{1}, x_{2}, x_{4}\right)=x_{3}$, and $T\left(x_{4}, x_{1}, x_{2}, x_{3}\right)=x_{4}$. 
Definition 2.12 ([20]). Let $X$ be a non-empty set, $T: X \times X \rightarrow X$ and $A: X \rightarrow X$ be two mappings. $A$ is said to be commutative with $T$, if $A T(x, y)=T(A x, A y)$ for all $x, y \in X$. A point $u \in X$ is called a common fixed point of $T$ and $A$, if $u=A u=T(u, u)$.

Definition 2.13. Let $X$ be a non-empty set, $T: X \times X \times X \times X \rightarrow X$ and $A: X \rightarrow X$ be two mappings. $A$ is said to be commutative with $T$, if $A T(x, y, p, q)=T(A x, A y, A p, A q)$ for all $x, y, p, q \in X$. A point $u \in X$ is called a common fixed point of $T$ and $A$, if $u=A u=T(u, u, u, u)$.

\section{Common fixed point results for probabilistic $\varphi$-contractions in generalized probabilistic metric spaces}

Theorem 3.1. Let $\left(\mathrm{X}, \mathrm{G}^{*}, \Delta\right)$ be a complete PGM-space such that $\Delta$ is a $\mathrm{t}$-norm of $\mathrm{H}$-type. Let $\varphi: \mathbb{R}^{+} \rightarrow \mathbb{R}^{+}$be a gauge function such that $\varphi^{-1}(\{0\})=\{0\}, \varphi(t)<\mathrm{t}$ and $\lim _{\mathrm{n} \rightarrow \infty} \varphi^{\mathrm{n}}(\mathrm{t})=0$ for any $\mathrm{t}>0$. Let $\mathrm{T}: \mathrm{X} \rightarrow \mathrm{X}$ and $\mathrm{g}: \mathrm{X} \rightarrow \mathrm{X}$ be two mappings such that

$$
\mathrm{G}_{\mathrm{T} x, T y, T z}^{*}(\varphi(t)) \geqslant \mathrm{G}_{A x, A y, A z}^{*}(t) G_{A y, A z, A z}^{*}(t) G_{A z, A y, A y}^{*}(t)
$$

for all $x, y, z \in X$, where $T(X) \subset A(X), A$ is continuous and commutative with $T$. Then there is a unique $u \in X$ such that $\mathrm{Au}=\mathrm{Tu}=\mathrm{u}$.

Proof. Let $x_{0} \in X$. By assumption $T(X) \subset A(X)$, there exists $x_{1} \in X$ such that $T x_{0}=A x_{1}$. By the same arguments, there exists $x_{2} \in X$ such that $T x_{1}=A x_{2}$. Continuing this process we can construct a sequence $\left\{x_{n}\right\}$ in $X$ such that

$$
A x_{n+1}=T x_{n}, \quad n \in \mathbb{N} .
$$

Due to (3.1), we have

$$
\begin{aligned}
\mathrm{G}_{A x_{n}, A x_{n+1}, A x_{n+1}}^{*}(\varphi(t))=G_{T x_{n-1}, T x_{n}, T x_{n}}^{*}(\varphi(t)) & \geqslant G_{A x_{n-1}, A x_{n}, A x_{n}}^{*}(t) G_{A x_{n}, A x_{n}, A x_{n}}^{*}(t) G_{A x_{n}, A x_{n}, A x_{n}}^{*}(t) \\
& =G_{A x_{n-1}, A x_{n}, A x_{n}}^{*}(t),
\end{aligned}
$$

by taking $x=x_{n-1}$ and $y=z=x_{n}$. Thus, for each natural number $n$, we have

$$
\mathrm{G}_{A x_{n}, A x_{n+1}, A x_{n+1}}^{*}\left(\varphi^{n}(t)\right) \geqslant G_{A x_{n-1}, A x_{n}, A x_{n}}^{*}\left(\varphi^{n-1}(t)\right) \geqslant \cdots \geqslant G_{A x_{1}, A x_{2}, A x_{2}}^{*}(\varphi(t)) \geqslant G_{A x_{0}, A x_{1}, A x_{1}}^{*}(t) .
$$

From Lemma 2.10, we have

$$
\lim _{n \rightarrow \infty} G_{A x_{n}, A x_{n+1}, A x_{n+1}}^{*}(t)=1
$$

for all $t>0$. For any $k \in \mathbb{Z}^{+}$and $t>0$, we shall show the following inequality by mathematical induction:

$$
G_{A x_{n}, A x_{n+k}, A x_{n+k}}^{*}(t) \geqslant \Delta^{k}\left(G_{A x_{n}, A x_{n+1}, A x_{n+1}}^{*}(t-\varphi(t))\right) \text {. }
$$

Let $k=1$. Since

$$
\begin{aligned}
\mathrm{G}_{A x_{n}, A x_{n+1}, A x_{n+1}}^{*}(t) & \geqslant G_{A x_{n}, A x_{n+1}, A x_{n+1}}^{*}(t-\varphi(t)) \\
& =\Delta\left(G_{A x_{n}, A x_{n+1}, A x_{n+1}}^{*}(t-\varphi(t)), 1\right) \\
& \geqslant \Delta\left(G_{A x_{n}, A x_{n+1}, A x_{n+1}}^{*}(t-\varphi(t)), G_{A x_{n}, A x_{n+1}, A x_{n+1}}^{*}(t-\varphi(t))\right) \\
& =\Delta^{1}\left(G_{A x_{n}, A x_{n+1}, A x_{n+1}}^{*}(t-\varphi(t))\right) .
\end{aligned}
$$

Thus (3.3) holds for $k=1$.

Suppose now that (3.3) holds for some fixed $k \geqslant 1$. Then, by the monotony of $G^{*}$ and (3.1) we get

$$
\begin{aligned}
G_{A x_{n+1}, A x_{n+k+1}, A x_{n+k+1}}^{*}(t) & \geqslant G_{A x_{n+1}, A x_{n+k+1}, A x_{n+k+1}}^{*}(\varphi(t)) \\
& =G_{T x_{n}, T x_{n+k}, T x_{n+k}}^{*}(\varphi(t)) \\
& \geqslant G_{A x_{n}, A x_{n+k}, A x_{n+k}}^{*}(t) \geqslant \Delta^{k}\left(G_{A x_{n}, A x_{n+1}, A x_{n+1}}^{*}(t-\varphi(t))\right) .
\end{aligned}
$$


Therefore, from (PGM-4) and (3.4), we have

$$
\begin{aligned}
\mathrm{G}_{A x_{n}, A x_{n+k+1}, A x_{n+k+1}}^{*}(t) & =G_{A x_{n}, A x_{n+k+1}, A x_{n+k+1}}^{*}(t-\varphi(t)+\varphi(t)) \\
& \geqslant \Delta\left(G_{A x_{n}, A x_{n+1}, A x_{n+1}}^{*}(t-\varphi(t)), G_{A x_{n+1}, A x_{n+k+1}, A x_{n+k+1}}^{*}(\varphi(t))\right) \\
& \geqslant \Delta\left(G_{A x_{n}, A x_{n+1}, A x_{n+1}}^{*}(t-\varphi(t)), \Delta^{k}\left(G_{A x_{n}, A x_{n+1}, A x_{n+1}}^{*}(t-\varphi(t))\right)\right. \\
& =\Delta^{k+1}\left(G_{A x_{n}, A x_{n+1}, A x_{n+1}}^{*}(t-\varphi(t))\right) .
\end{aligned}
$$

Thus we prove that if (3.3) holds for some fixed $k \geqslant 1$, then (3.3) holds for $k+1$. Then by the mathematical induction we conclude that (3.3) holds for all $k \geqslant 1$.

Next, we shall prove that $\left\{A x_{n}\right\}$ is a Cauchy sequence, that is,

$$
\lim _{m, n, l \rightarrow \infty} G_{A x_{n}, A x_{m}, A x_{l}}^{*}(t)=1
$$

for any $t>0$. For this aim, firstly, we can show that $\lim _{m, n, \rightarrow \infty} G_{A x_{n}, A x_{m}, A x_{m}}^{*}(t)=1$ for any $t>0$. Suppose that $\varepsilon \in(0,1]$ is given. Since $\Delta$ is a t-norm of H-type, there exists $\delta>0$, such that

$$
\Delta^{\mathrm{n}}(\mathrm{s})>1-\varepsilon
$$

for all $n \geqslant 1$ and when $1-\delta<s \leqslant 1$.

On the other hand, from (3.2) we have

$$
\lim _{n \rightarrow \infty} G_{A x_{n}, A x_{n+1}, A x_{n+1}}^{*}(t-\varphi(t))=1,
$$

which implies that there exists $n_{0} \in \mathbb{N}$ such that $G_{A x_{n}, A x_{n+1}, A x_{n+1}}^{*}(t-\varphi(t))>1-\delta$ for all $n \geqslant n_{0}$. Hence, using (3.3) and (3.5), we obtain that $G_{A x_{n}, A x_{n+k}, A x_{n+k}}^{*}(t)>1-\epsilon$ for $k \in \mathbb{Z}^{+}$and $n \geqslant n_{0}$. Thus

$$
\lim _{m, n \rightarrow \infty} G_{A x_{n}, A x_{m}, A x_{m}}^{*}(t)=1
$$

for any $t>0$. Moreover, from (PGM-4), we get that

$$
\begin{gathered}
\mathrm{G}_{A x_{n}, A x_{m}, A x_{l}}^{*}(t) \geqslant \Delta\left(G_{A x_{n}, A x_{n}, A x_{m}}^{*}\left(\frac{t}{2}\right), G_{A x_{n}, A x_{n}, A x_{l}}^{*}\left(\frac{t}{2}\right)\right), \\
G_{A x_{n}, A x_{n}, A x_{m}}^{*}\left(\frac{t}{2}\right) \geqslant \Delta\left(G_{A x_{n}, A x_{m}, A x_{m}}^{*}\left(\frac{t}{4}\right), G_{A x_{n}, A x_{m}, A x_{m}}^{*}\left(\frac{t}{4}\right)\right),
\end{gathered}
$$

and

$$
G_{A x_{n}, A x_{n}, A x_{l}}^{*}\left(\frac{t}{2}\right) \geqslant \Delta\left(G_{A x_{n}, A x_{l}, A x_{l}}^{*}\left(\frac{t}{4}\right), G_{A x_{n}, A x_{l}, A x_{l}}^{*}\left(\frac{t}{4}\right)\right)
$$

Therefore, by the continuity of $\Delta$, we have

$$
\lim _{m, n, l \rightarrow \infty} G_{A x_{n}, A x_{m}, A x_{l}}^{*}(t)=1
$$

for any $t>0$. This means that $\left\{A x_{n}\right\}$ is a Cauchy sequence. Since $X$ is complete, then there exists $u \in X$ such that $\lim _{n \rightarrow \infty}\left\{A x_{n}\right\}=u$. Since $A$ is continuous, we have $\lim _{n \rightarrow \infty}\left\{A A x_{n}\right\}=A u$. On the other hand, we have $A A x_{n+1}=A T x_{n}=T A x_{n}$ since $A$ and $T$ commute. Thus, from (3.1) we have

$$
\mathrm{G}_{A x_{\mathfrak{n}+1}, T u, T u}^{*}(\varphi(t))=\mathrm{G}_{T A x_{n}, T u, T u}^{*}(\varphi(t)) \geqslant G_{A A x_{n}, A u, A u}^{*}(t) .
$$

The sequence $\left\{A x_{n+1}\right\}$ is convergent to $u$ since $\left\{A x_{n+1}\right\}$ is a subsequence of $\left\{A x_{n}\right\}$. Letting $n \rightarrow \infty$ on both sides of inequality (3.6), we get that

$$
\mathrm{G}_{\mathrm{Au}, \mathrm{Tu}, \mathrm{Tu}}^{*}(\varphi(t)) \geqslant \mathrm{G}_{\mathrm{Au}, A u, A u}^{*}(t) .
$$


Hence $A u=T u$.

Next, we shall prove that $u=A u=T u$. By (3.1) we obtain that

$$
\mathrm{G}_{A x_{n}, A u, A u}^{*}(\varphi(t))=\mathrm{G}_{A x_{n}, T u, T u}^{*}(\varphi(t))=G_{T x_{n-1}, T u, T u}^{*}(\varphi(t)) \geqslant G_{A x_{n-1}, A u, A u}^{*}(t) .
$$

Denote $B_{n}(t)=G_{A x_{n}, A u, A u}^{*}(t)$. By (3.7), it is easy to find that

$$
\mathrm{B}_{\mathrm{n}}\left(\varphi^{\mathrm{n}}(\mathrm{t})\right) \geqslant \mathrm{B}_{\mathrm{n}-1}\left(\varphi^{\mathrm{n}-1}(\mathrm{t})\right) \geqslant \cdots \geqslant \mathrm{B}_{1}(\varphi(\mathrm{t})) \geqslant \mathrm{B}_{0}(\mathrm{t}) .
$$

Since $\mathrm{B}_{0}(\mathrm{t}) \in \mathcal{D}^{+}$and $\lim _{n \rightarrow \infty} \varphi^{\mathrm{n}}(\mathrm{t})=0$, by Lemma 2.10 we get

$$
\lim _{n \rightarrow \infty} B_{n}(t)=\lim _{n \rightarrow \infty} G_{A x_{n}, A u, A u}^{*}(t)=1,
$$

which implies that $u=A u$. Then $u=A u=T u$.

Now we show that $u$ is the unique common fixed point of $T$ and $A$. Suppose that, contrary to our claim, there exists another common fixed point $w \in X$ with $w \neq u$. From (3.1) we have

$$
\mathrm{G}_{w, u, u}^{*}(\varphi(t))=\mathrm{G}_{\mathrm{T} w, \mathrm{Tu}, \mathrm{Tu}}^{*}(\varphi(\mathrm{t})) \geqslant \mathrm{G}_{\mathrm{A} w, \mathrm{Au}, \mathrm{Au}}^{*}(\mathrm{t})=\mathrm{G}_{w, \mathrm{u}, \mathrm{u}}^{*}(\mathrm{t}),
$$

which is a contradiction since $\varphi(t)<t$. Hence, the common fixed point of $T$ and $A$ is unique.

Corollary 3.2. Let $\left(\mathrm{X}, \mathrm{G}^{*}, \Delta\right)$ be a complete PGM-space such that $\Delta$ is a $\mathrm{t}$-norm of $\mathrm{H}$-type. Let $\varphi: \mathbb{R}^{+} \rightarrow \mathbb{R}^{+}$be a gauge function such that $\varphi^{-1}(\{0\})=\{0\}, \varphi(\mathrm{t})<\mathrm{t}$ and $\lim _{\mathrm{n} \rightarrow \infty} \varphi^{\mathrm{n}}(\mathrm{t})=0$ for any $\mathrm{t}>0$. Let $\mathrm{T}: \mathrm{X} \rightarrow \mathrm{X}$ and $\mathrm{g}: \mathrm{X} \rightarrow \mathrm{X}$ be two mappings such that

$$
\mathrm{G}_{\mathrm{T} x, \mathrm{Ty}, \mathrm{Tz}}^{*}(\varphi(\mathrm{t})) \geqslant \mathrm{G}_{\mathrm{Ax}, \mathrm{Ay}, \mathrm{Az}}^{*}(\mathrm{t})
$$

for all $x, y, z \in X$, where $\mathrm{T}(\mathrm{X}) \subset \mathrm{A}(\mathrm{X}), \mathrm{A}$ is continuous and commutative with $\mathrm{T}$. Then there is a unique $\mathrm{u} \in \mathrm{X}$ such that $\mathrm{Au}=\mathrm{Tu}=\mathrm{u}$.

Proof. Due to

$$
G_{T x, T y, T z}^{*}(\varphi(t)) \geqslant G_{A x, A y, A z}^{*}(t) \geqslant G_{A x, A y, A z}^{*}(t) G_{A y, A z, A z}^{*}(t) G_{A z, A y, A y}^{*}(t),
$$

we conclude from Theorem 3.1 that the mappings $T$ and $A$ have a unique common fixed point in $X$.

Remark 3.3. Corollary 3.2 is the probabilistic version of Theorem 3.1 in [1].

Taking $y=z$ in Corollary 3.2, we get the following corollary.

Corollary 3.4. Let $\left(\mathrm{X}, \mathrm{G}^{*}, \Delta\right)$ be a complete PGM-space such that $\Delta$ is a t-norm of $\mathrm{H}$-type. Let $\varphi: \mathbb{R}^{+} \rightarrow \mathbb{R}^{+}$be a gauge function such that $\varphi^{-1}(\{0\})=\{0\}, \varphi(t)<\mathrm{t}$ and $\lim _{\mathrm{n} \rightarrow \infty} \varphi^{\mathrm{n}}(\mathrm{t})=0$ for any $\mathrm{t}>0$. Let $\mathrm{T}: \mathrm{X} \rightarrow \mathrm{X}$ and $\mathrm{A}: \mathrm{X} \rightarrow \mathrm{X}$ be two mappings such that

$$
\mathrm{G}_{\mathrm{T} x, T y, T y}^{*}(\varphi(t)) \geqslant \mathrm{G}_{A x, A y, A y}^{*}(\mathrm{t})
$$

for all $x, y \in X$, where $T(X) \subset A(X), A$ is continuous and commutative with $T$. Then there is a unique $u \in X$ such that $\mathrm{Au}=\mathrm{Tu}=\mathrm{u}$.

Remark 3.5. Corollary 3.4 is the probabilistic version of Theorem 3.2 in [1].

In Theorem 3.1, if we take $A=I$ ( $\mathrm{I}$ is the identity mapping), then we have the following result.

Corollary 3.6. Let $\left(\mathrm{X}, \mathrm{G}^{*}, \Delta\right)$ be a complete PGM-space such that $\Delta$ is a $\mathrm{t}$-norm of $\mathrm{H}$-type. Let $\varphi: \mathbb{R}^{+} \rightarrow \mathbb{R}^{+}$be a gauge function such that $\varphi^{-1}(\{0\})=\{0\}, \varphi(\mathrm{t})<\mathrm{t}$ and $\lim _{\mathrm{n} \rightarrow \infty} \varphi^{\mathrm{n}}(\mathrm{t})=0$ for any $\mathrm{t}>0$. Let $\mathrm{T}: \mathrm{X} \rightarrow \mathrm{X}$ be a mapping such that

$$
\mathrm{G}_{T x, T y, T z}^{*}(\varphi(t)) \geqslant \mathrm{G}_{x, y, z}^{*}(\mathrm{t}) \mathrm{G}_{y, z, z}^{*}(\mathrm{t}) \mathrm{G}_{z, y, y}^{*}(\mathrm{t})
$$

for all $\mathrm{x}, \mathrm{y}, z \in \mathrm{X}$ and $\mathrm{t}>0$. Then $\mathrm{T}$ has a unique fixed point in $\mathrm{X}$. 
In Theorem 3.1, if we take $\varphi(t)=\lambda t(0<\lambda<1)$, then we have the following corollary.

Corollary 3.7. Let $\left(\mathrm{X}, \mathrm{G}^{*}, \Delta\right)$ be a complete PGM-space such that $\Delta$ is a $\mathrm{t}$-norm of $\mathrm{H}$-type. Let $\mathrm{T}: \mathrm{X} \rightarrow \mathrm{X}$ and $A: X \rightarrow X$ be two mappings such that

$$
G_{T x, T y, T z}^{*}(\lambda t) \geqslant G_{A x, A y, A z}^{*}(t) G_{A y, A z, A z}^{*}(t) G_{A z, A y, A y}^{*}(t)
$$

for all $x, y \in X$, where $\mathrm{T}(\mathrm{X}) \subset \mathrm{A}(\mathrm{X}), \mathrm{A}$ is continuous and commutative with $\mathrm{T}$. Then there is a unique $\mathrm{u} \in \mathrm{X}$ such that $\mathrm{Au}=\mathrm{Tu}=\mathrm{u}$.

Following similar argument in the proof of Theorem 3.1, we can deduce the next theorem. We omit the details of the proof.

Theorem 3.8. Let $\left(\mathrm{X}, \mathrm{G}^{*}, \Delta\right)$ be a complete PGM-space such that $\Delta$ is a $\mathrm{t}$-norm of $\mathrm{H}$-type. Let $\varphi: \mathbb{R}^{+} \rightarrow \mathbb{R}^{+}$be a gauge function such that $\varphi^{-1}(\{0\})=\{0\}, \varphi(t)>t$, and $\lim _{n \rightarrow \infty} \varphi^{n}(t)=+\infty$ for any $\mathrm{t}>0$. Let $\mathrm{T}: \mathrm{X} \rightarrow \mathrm{X}$ and $\mathrm{A}: \mathrm{X} \rightarrow \mathrm{X}$ be two mappings such that

$$
\mathrm{G}_{\mathrm{T} x, T y, T z}^{*}(t) \geqslant \mathrm{G}_{A x, A y, A z}^{*}(\varphi(t)) \mathrm{G}_{\mathrm{Ay}, A z, A z}^{*}(\varphi(t)) \mathrm{G}_{A z, A y, A y}^{*}(\varphi(t))
$$

for all $x, y, z \in X$, where $T(X) \subset A(X), A$ is continuous and commutative with $T$. Then there is a unique $u \in X$ such that $\mathrm{Au}=\mathrm{Tu}=\mathrm{u}$.

Taking $y=z$ in Theorem 3.8, we get the following corollary.

Corollary 3.9. Let $\left(\mathrm{X}, \mathrm{G}^{*}, \Delta\right)$ be a complete PGM-space such that $\Delta$ is a $\mathrm{t}$-norm of $\mathrm{H}$-type. Let $\varphi: \mathbb{R}^{+} \rightarrow \mathbb{R}^{+}$be a gauge function such that $\varphi^{-1}(\{0\})=\{0\}, \varphi(t)>t$ and $\lim _{n \rightarrow \infty} \varphi^{n}(t)=+\infty$ for any $t>0$. Let $\mathrm{T}: X \rightarrow X$ and $A: X \rightarrow X$ be two mappings such that

$$
\mathrm{G}_{T x, T y, T y}^{*}(t) \geqslant \mathrm{G}_{A x, A y, A y}^{*}(\varphi(t))
$$

for all $x, y \in X$, where $\mathrm{T}(\mathrm{X}) \subset \mathrm{A}(\mathrm{X}), \mathrm{A}$ is continuous and commutative with $\mathrm{T}$. Then there is a unique $\mathrm{u} \in \mathrm{X}$ such that $\mathrm{Au}=\mathrm{Tu}=\mathrm{u}$.

In Theorem 3.8, if we take $A=I$ ( $I$ is the identity mapping), then we have the following corollary.

Corollary 3.10. Let $\left(\mathrm{X}, \mathrm{G}^{*}, \Delta\right)$ be a complete PGM-space such that $\Delta$ is a $\mathrm{t}$-norm of $\mathrm{H}$-type. Let $\varphi: \mathbb{R}^{+} \rightarrow \mathbb{R}^{+}$be a gauge function such that $\varphi^{-1}(\{0\})=\{0\}, \varphi(\mathrm{t})>\mathrm{t}$ and $\lim _{\mathrm{n} \rightarrow \infty} \varphi^{\mathrm{n}}(\mathrm{t})=+\infty$ for any $\mathrm{t}>0$. Let $\mathrm{T}: \mathrm{X} \rightarrow \mathrm{X}$ be a mapping such that

$$
\mathrm{G}_{\mathrm{T} x, T y, T z}^{*}(\mathrm{t}) \geqslant \mathrm{G}_{x, y, z}^{*}(\varphi(t)) \mathrm{G}_{y, z, z}^{*}(\varphi(t)) \mathrm{G}_{z, y, y}^{*}(\varphi(t))
$$

for all $\mathrm{x}, \mathrm{y}, z \in \mathrm{X}$ and $\mathrm{t}>0$. Then $\mathrm{T}$ has a unique fixed point in $\mathrm{X}$.

Next, we present some new common coupled fixed point results under probabilistic $\varphi$-contractive conditions in PGM-space.

Theorem 3.11. Let $\left(\mathrm{X}, \mathrm{G}^{*}, \Delta\right)$ be a complete PGM-space such that $\Delta$ is a $\mathrm{t}$-norm of $\mathrm{H}$-type. Let $\varphi: \mathbb{R}^{+} \rightarrow \mathbb{R}^{+}$be a gauge function such that $\varphi^{-1}(\{0\})=\{0\}, \varphi(t)<\mathrm{t}$ and $\lim _{\mathrm{n} \rightarrow \infty} \varphi^{\mathrm{n}}(\mathrm{t})=0$ for any $\mathrm{t}>0$. Let $\mathrm{T}: \mathrm{X} \times \mathrm{X} \rightarrow \mathrm{X}$ and $\mathrm{A}: \mathrm{X} \rightarrow \mathrm{X}$ be two mappings such that

$$
\begin{aligned}
G_{T}^{*}(x, y), T(p, q), T(r, s) & (\varphi(t)) \geqslant \\
& {\left[G_{A x, A p, A r}^{*}(t) G_{A y, A q, A s}^{*}(t) G_{A x, A p, A p}^{*}(t) G_{A y, A q, A q}^{*}(t)\right.} \\
& \left.\times G_{A p, A r, A r}^{*}(t) G_{A q, A s, A s}^{*}(t) G_{A x, A r, A r}^{*}(t) G_{A y, A s, A s}^{*}(t)\right]^{\frac{1}{6}}
\end{aligned}
$$

for all $x, y, p, q, r, s \in X$, where $T(X \times X) \subset A(X), A$ is continuous and commutative with $T$. Then there exists a unique $\mathrm{u} \in \mathrm{X}$ such that $\mathrm{u}=\mathrm{Au}=\mathrm{T}(\mathrm{u}, \mathrm{u})$. 
Proof. Let $x_{0}, y_{0}$ be two arbitrary points of $X$. Since $T(X \times X) \subset A(X)$, we can choose $x_{1}, y_{1} \in X$ such that $A x_{1}=T\left(x_{0}, y_{0}\right)$ and $A y_{1}=T\left(y_{0}, x_{0}\right)$. Again from $T(X \times X) \subset A(X)$, we can choose $x_{2}, y_{2} \in X$ such that $A x_{2}=T\left(x_{1}, y_{1}\right)$ and $A y_{2}=T\left(y_{1}, x_{1}\right)$. Continuing this process we can construct sequences $\left\{x_{n}\right\}$ and $\left\{y_{n}\right\}$ in $X$ such that

$$
A x_{n+1}=T\left(x_{n}, y_{n}\right) \text { and } A y_{n+1}=T\left(y_{n}, x_{n}\right)
$$

for all $n \in \mathbb{N}$. From (3.8), we get

$$
\begin{aligned}
\mathrm{G}_{A x_{n}, A x_{n+1}, A x_{n+1}}^{*}(\varphi(t)) & =\mathrm{G}_{\mathrm{T}\left(x_{n-1}, y_{n-1}\right), T\left(x_{n}, y_{n}\right), T\left(x_{n}, y_{n}\right)}^{*}(\varphi(t)) \\
& \geqslant\left[G_{A x_{n-1}, A x_{n}, A x_{n}}^{*}(t) G_{A y_{n-1}, A y_{n}, A y_{n}}^{*}(t)\right]^{1 / 2}
\end{aligned}
$$

and

$$
\begin{aligned}
\mathrm{G}_{A y_{n}, A y_{n+1}, A y_{n+1}}^{*}(\varphi(t)) & =G_{T\left(y_{n-1}, x_{n-1}\right), T\left(y_{n}, x_{n}\right), T\left(y_{n}, x_{n}\right)}^{*}(\varphi(t)) \\
& \geqslant\left[G_{A y_{n-1}, A y_{n}, A y_{n}}^{*}(t) G_{A x_{n-1}, A x_{n}, A x_{n}}^{*}(t)\right]^{1 / 2}
\end{aligned}
$$

for any $t>0$.

To simplify the writing, denote $P_{n}(t)=\left[G_{A x_{n-1}, A x_{n}, A x_{n}}^{*}(t) G_{A y_{n-1}, A y_{n}, A y_{n}}^{*}(t)\right]^{1 / 2}$. Using (3.9) and (3.10) we obtain that $P_{n+1}(\varphi(t)) \geqslant P_{n}(t)$. Hence, for $n \geqslant 1$ it follows that

$$
\mathrm{G}_{A x_{n}, A x_{n+1}, A x_{n+1}}^{*}\left(\varphi^{n}(t)\right) \geqslant P_{n}\left(\varphi^{n-1}(t)\right) \geqslant \cdots \geqslant P_{1}(t)
$$

and

$$
G_{A y_{n}, A y_{n+1}, A y_{n+1}}^{*}\left(\varphi^{n}(t)\right) \geqslant P_{n}\left(\varphi^{n-1}(t)\right) \geqslant \cdots \geqslant P_{1}(t)
$$

Since $P_{1}(t)=\left[G_{A x_{0}, A x_{1}, A x_{1}}^{*}(t) G_{A y_{0}, A y_{1}, A y_{1}}^{*}(t)\right]^{1 / 2} \in \mathcal{D}^{+}$and $\lim _{n \rightarrow \infty} \varphi^{n}(t)=0$ for any $t>0$, by Lemma 2.10 we get

$$
\lim _{n \rightarrow \infty} G_{A x_{n}, A x_{n+1}, A x_{n+1}}^{*}(t)=1
$$

and

$$
\lim _{n \rightarrow \infty} G_{A y_{n}, A y_{n+1}, A y_{n+1}}^{*}(t)=1
$$

Thus, by (3.11) and (3.12), we obtain that

$$
\lim _{n \rightarrow \infty} P_{n}(t)=1
$$

for all $t>0$.

Now we prove by induction that

$$
G_{A x_{n}, A x_{n+k}, A x_{n+k}}^{*}(t) \geqslant \Delta^{k}\left(P_{n}(t-\varphi(t))\right)
$$

and

$$
G_{A y_{n}, A y_{n+k}, A y_{n+k}}^{*}(t) \geqslant \Delta^{k}\left(P_{n}(t-\varphi(t))\right)
$$

for any $k \in \mathbb{Z}^{+}$.

Indeed, if we take $k=1$, then from the monotonicity of $\mathrm{G}^{*}$ and (3.8), we obtain that

$$
\begin{aligned}
G_{A x_{n}, A x_{n+1}, A x_{n+1}}^{*}(t) & \geqslant G_{A x_{n}, A x_{n+1}, A x_{n+1}}^{*}(\varphi(t)) \\
& =G_{T\left(x_{n-1}, y_{n-1}\right), T\left(x_{n}, y_{n}\right), T\left(x_{n}, y_{n}\right)}^{*}(\varphi(t)) \\
& \geqslant\left[G_{A x_{n-1}, A x_{n}, A x_{n}}^{*}(t) G_{A y_{n-1}, A y_{n}, A y_{n}}^{*}(t)\right]^{\frac{1}{2}} \\
& \geqslant\left[G_{A x_{n-1}, A x_{n}, A x_{n}}^{*}(t-\varphi(t)) G_{A y_{n-1}, A y_{n}, A y_{n}}^{*}(t-\varphi(t))\right]^{\frac{1}{2}} \\
& =P_{n}(t-\varphi(t)) \\
& =\Delta\left(P_{n}(t-\varphi(t)), 1\right) \\
& \geqslant \Delta\left(P_{n}(t-\varphi(t)), P_{n}(t-\varphi(t))\right) \\
& =\Delta^{1}\left(P_{n}(t-\varphi(t))\right) .
\end{aligned}
$$


Similarly, we have

$$
\mathrm{G}_{A y_{n}, A y_{n+1}, A y_{n+1}}^{*}(t) \geqslant \Delta^{1}\left(P_{n}(t-\varphi(t))\right) .
$$

Thus (3.13) and (3.14) hold for $k=1$.

If we assume that (3.13) is true for some fixed $k$, then since $\varphi(t)<t$, by (3.9) we have

$$
G_{A x_{n}, A x_{n+1}, A x_{n+1}}^{*}(t) \geqslant G_{A x_{n}, A x_{n+1}, A x_{n+1}}^{*}(\varphi(t)) \geqslant P_{n}(t)
$$

for all $t>0$. Moreover, from (3.8) and (3.13), we get

$$
\begin{aligned}
G_{A x_{n+1}, A x_{n+k+1}, A x_{n+k+1}}^{*}(\varphi(t)) & \geqslant\left[G_{A x_{n}, A x_{n+k}, A x_{n+k}}^{*}(t) G_{A y_{n}, A y_{n+k}, A y_{n+k}}^{*}(t)\right]^{1 / 2} \\
& \geqslant \Delta^{k}\left(P_{n}(t-\varphi(t))\right) .
\end{aligned}
$$

Hence, by (PGM-4), (3.15), (3.16), and the monotonicity of $\Delta$, we obtain that

$$
\begin{aligned}
\mathrm{G}_{A x_{n}, A x_{n+k+1}, A x_{n+k+1}}^{*}(t) & =G_{A x_{n}, A x_{n+k+1}, A x_{n+k+1}}^{*}(t-\varphi(t)+\varphi(t)) \\
& \geqslant \Delta\left(G_{A x_{n}, A x_{n+1}, A x_{n+1}}^{*}(t-\varphi(t)), G_{A x_{n+1}, A x_{n+k+1}, A x_{n+k+1}}^{*}(\varphi(t))\right) \\
& \geqslant \Delta\left(P_{n}(t-\varphi(t)), \Delta^{k}\left(P_{n}(t-\varphi(t))\right)\right)=\Delta^{k+1}\left(P_{n}(t-\varphi(t))\right) .
\end{aligned}
$$

Similarly, we have $G_{A y_{n}, A y_{n+k+1}, A y_{n+k+1}}^{*}(t) \geqslant \Delta^{k+1}\left(P_{n}(t-\varphi(t))\right)$. Therefore, by induction, (3.13) and (3.14) hold for all $k \in \mathbb{Z}^{+}$.

Next, we shall prove that $\left\{A x_{n}\right\}$ and $\left\{A y_{n}\right\}$ are all Cauchy sequences, that is,

$$
\lim _{m, n, l \rightarrow \infty} G_{A x_{n}, A x_{m}, A x_{l}}^{*}(t)=1 \text { and } \lim _{m, n, l \rightarrow \infty} G_{A y_{n}, A y_{m}, A y_{l}}^{*}(t)=1
$$

for any $t>0$.

To this end, firstly, we can show that

$$
\lim _{m, n \rightarrow \infty} G_{A x_{n}, A x_{m}, A x_{m}}^{*}(t)=1 \text { and } \lim _{m, n \rightarrow \infty} G_{A y_{n}, A y_{m}, A y_{m}}^{*}(t)=1
$$

for any $t>0$.

Suppose that $\varepsilon \in(0,1]$ is given. Since $\Delta$ is a t-norm of H-type, there exists $\delta>0$, such that

$$
\Delta^{\mathrm{n}}(\mathrm{s})>1-\varepsilon
$$

for all $n \geqslant 1$ and when $1-\delta<s \leqslant 1$.

On the other hand, by (3.11) and (3.12), we have

$$
\lim _{n \rightarrow \infty} G_{A x_{n}, A x_{n+1}, A x_{n+1}}^{*}(t-\varphi(t))=1 \quad \text { and } \quad \lim _{n \rightarrow \infty} G_{A y_{n}, A y_{n+1}, A y_{n+1}}^{*}(t-\varphi(t))=1
$$

That is

$$
\lim _{n \rightarrow \infty} P_{n}(t-\varphi(t))=1,
$$

which implies that there exists $n_{0} \in \mathbb{N}$ such that $P_{n}(t-\varphi(t))>1-\delta$ for all $n \geqslant n_{0}$. Hence, from (3.13), (3.14), and (3.17), we get

$$
G_{A x_{n}, A x_{n+k}, A x_{n+k}}^{*}(t)>1-\epsilon \text { and } G_{A y_{n}, A y_{n+k}, A y_{n+k}}^{*}(t)>1-\epsilon
$$

for $k \in \mathbb{Z}^{+}$and $n \geqslant n_{0}$. These show that

$$
\lim _{m, n \rightarrow \infty} G_{A x_{n}, A x_{m}, A x_{m}}^{*}(t)=1 \text { and } \lim _{m, n \rightarrow \infty} G_{A y_{n}, A y_{m}, A y_{m}}^{*}(t)=1
$$


for any $t>0$. From (PGM-4), it follows that

$$
\begin{aligned}
G_{A x_{n}, A x_{m}, A x_{l}}^{*}(t) & \geqslant \Delta\left(G_{A x_{n}, A x_{n}, A x_{m}}^{*}\left(\frac{t}{3}\right), G_{A x_{n}, A x_{n}, A x_{l}}^{*}\left(\frac{2 t}{3}\right)\right) ; \\
G_{A x_{n}, A x_{n}, A x_{m}}^{*}\left(\frac{t}{3}\right) & \geqslant \Delta\left(G_{A x_{n}, A x_{m}, A x_{m}}^{*}\left(\frac{t}{6}\right), G_{A x_{n}, A x_{m}, A x_{m}}^{*}\left(\frac{t}{6}\right)\right) ; \\
G_{A x_{n}, A x_{n}, A x_{l}}^{*}\left(\frac{2 t}{3}\right) & \geqslant \Delta\left(G_{A x_{n}, A x_{l}, A x_{l}}^{*}\left(\frac{t}{3}\right), G_{A x_{n}, A x_{l}, A x_{l}}^{*}\left(\frac{t}{3}\right)\right) .
\end{aligned}
$$

Therefore, by the continuity of $\Delta$, we have

$$
\lim _{m, n, l \rightarrow \infty} G_{A x_{n}, A x_{m}, A x_{l}}^{*}(t)=1 \text { and } \lim _{m, n, l \rightarrow \infty} G_{A y_{n}, A y_{m}, A y_{l}}^{*}(t)=1
$$

for any $t>0$. These imply that $\left\{A x_{n}\right\}$ and $\left\{A y_{n}\right\}$ are all Cauchy sequences. Since $X$ is complete, there exist $u, v \in X$ such that $\lim _{n \rightarrow \infty} A x_{n}=u$ and $\lim _{n \rightarrow \infty} A y_{n}=v$. By the continuity of $A$, we get

$$
\lim _{n \rightarrow \infty} A A x_{n}=A u \text { and } \lim _{n \rightarrow \infty} A A y_{n}=A v
$$

Since $A$ and $T$ are commutative mappings, we obtain

$$
A A x_{n+1}=A T\left(x_{n}, y_{n}\right)=T\left(A x_{n}, A y_{n}\right) .
$$

Then, from (3.8) and $\varphi(t)<t$, we have

$$
\begin{aligned}
\mathrm{G}_{A A x_{n+1}, T(u, v), T(u, v)}^{*}(t) & \geqslant G_{A A x_{n+1}, T(u, v), T(u, v)}^{*}(\varphi(t)) \\
& =G_{T\left(A x_{n}, A y_{n}\right), T(u, v), T(u, v)}^{*}(\varphi(t)) \\
& \geqslant\left[G_{A A x_{n}, A u, A u}^{*}(t) G_{A A y_{n}, A v, A v}^{*}(t)\right]^{1 / 2} .
\end{aligned}
$$

Taking the limit as $n$ tends to infinity in (3.18), we obtain $\lim _{n \rightarrow \infty} A A x_{n}=T(u, v)$. Hence, $T(u, v)=A u$. In a similar way, we can prove that $T(v, u)=A v$.

Next, we shall show that $A u=v$ and $A v=u$. Indeed, from (3.8) we obtain that

$$
\begin{aligned}
\mathrm{G}_{\mathrm{Au}, A y_{n}, A y_{n}}^{*}(\varphi(t)) & =\mathrm{G}_{\mathrm{T}(u, v), T\left(y_{n-1}, x_{n-1}\right), T\left(y_{n-1}, x_{n-1}\right)}^{*}(\varphi(t)) \\
& \geqslant\left[G_{A u, A y_{n-1}, A y_{n-1}}^{*}(t) G_{A v, A x_{n-1}, A x_{n-1}}^{*}(t)\right]^{1 / 2} .
\end{aligned}
$$

Similarly, we have

$$
\mathrm{G}_{A v, A x_{n}, A x_{n}}^{*}(\varphi(t)) \geqslant\left[G_{A v, A x_{n-1}, A x_{n-1}}^{*}(t) G_{A u, A y_{n-1}, A y_{n-1}}^{*}(t)\right]^{1 / 2} .
$$

Denote $Q_{n}(t)=G_{A u, A y_{n}, A y_{n}}^{*}(t) G_{A v, A x_{n}, A x_{n}}^{*}(t)$. From (3.19) and (3.20), we get

$$
\begin{aligned}
\mathrm{Q}_{\mathrm{n}}\left(\varphi^{\mathrm{n}}(\mathrm{t})\right) & \geqslant \mathrm{Q}_{\mathrm{n}-1}\left(\varphi^{\mathrm{n}-1}(\mathrm{t})\right) \geqslant \cdots \geqslant \mathrm{Q}_{0}(\mathrm{t}), \\
\mathrm{G}_{\mathrm{Au}, \mathrm{A} \mathrm{y}_{\mathrm{n}}, \mathrm{A}_{\mathrm{n}}}^{*}\left(\varphi^{\mathrm{n}}(\mathrm{t})\right) & \geqslant\left[\mathrm{Q}_{0}(\mathrm{t})\right]^{1 / 2},
\end{aligned}
$$

and

$$
\mathrm{G}_{\mathrm{A} v, A x_{n}, A x_{n}}^{*}\left(\varphi^{\mathrm{n}}(\mathrm{t})\right) \geqslant\left[\mathrm{Q}_{0}(\mathrm{t})\right]^{1 / 2} .
$$

Since $\left[\mathrm{Q}_{0}(\mathrm{t})\right]^{1 / 2} \in \mathcal{D}^{+}$and $\lim _{\mathrm{n} \rightarrow \infty} \varphi^{\mathrm{n}}(\mathrm{t})=0$, by Lemma 2.10 we obtain that

$$
\lim _{n \rightarrow \infty} A y_{n}=A u \text { and } \lim _{n \rightarrow \infty} A x_{n}=A v .
$$


These show that $v=A u$ and $u=A v$. Hence, $v=T(u, v)$ and $u=T(v, u)$. Finally, we prove that $u=v$. By (3.8), we have

$$
\begin{aligned}
& \mathrm{G}_{u, v, u}^{*}(\varphi(t))=\mathrm{G}_{\mathrm{T}(v, u), T(u, v), T(v, u)}^{*}(\varphi(t)) \geqslant\left[\mathrm{G}_{A v, A u, A v}^{*}(t) G_{A u, A v, A u}^{*}(t)\right]^{1 / 2} \\
& =\left[\mathrm{G}_{\mathrm{u}, v, \mathrm{u}}^{*}(\mathrm{t}) \mathrm{G}_{v, \mathrm{u}, v}^{*}(\mathrm{t})\right]^{1 / 2}
\end{aligned}
$$

and

$$
\begin{aligned}
\mathrm{G}_{v, u, v}^{*}(\varphi(t))=\mathrm{G}_{\mathbf{T}(u, v), T(v, u), T(u, v)}^{*}(\varphi(t)) & \geqslant\left[\mathrm{G}_{A u, A v, A u}^{*}(t) G_{A v, A u, A v}^{*}(t)\right]^{1 / 2} \\
& =\left[G_{v, u, v}^{*}(t) G_{u, v, u}^{*}(t)\right]^{1 / 2} .
\end{aligned}
$$

Suppose that $R(t)=\left[G_{v, u, v}^{*}(t) G_{u, v, u}^{*}(t)\right]^{1 / 2}$. From (3.21) and (3.22), we have $R\left(\varphi^{n}(t)\right) \geqslant R(t)$. Using Lemma 2.10, we have $R(t)=1$, i.e., $u=v$. The uniqueness of $u$ follows from (3.8). This completes the proof.

Remark 3.12. Theorem 3.11 generalizes and extends the corresponding result in Xiao et al. [22].

In Theorem 3.11, if we take $A=I$ ( $\mathrm{I}$ is the identity mapping), then we have the following corollary.

Corollary 3.13. Let $\left(\mathrm{X}, \mathrm{G}^{*}, \Delta\right)$ be a complete PGM-space such that $\Delta$ is a $\mathrm{t}$-norm of $\mathrm{H}$-type. Let $\varphi: \mathbb{R}^{+} \rightarrow \mathbb{R}^{+}$be a gauge function such that $\varphi^{-1}(\{0\})=\{0\}, \varphi(\mathrm{t})<\mathrm{t}$ and $\lim _{\mathrm{n} \rightarrow \infty} \varphi^{\mathrm{n}}(\mathrm{t})=0$ for any $\mathrm{t}>0$. Let $\mathrm{T}: \mathrm{X} \times \mathrm{X} \rightarrow \mathrm{X}$ be a mapping satisfying

$$
\begin{aligned}
& \mathrm{G}_{\mathrm{T}(x, y), T(p, q), T(r, s)}^{*}(\varphi(t)) \geqslant\left[\mathrm{G}_{x, p, r}^{*}(t) G_{y, q, s}^{*}(t) G_{x, p, p}^{*}(t) G_{y, q, q}^{*}(t)\right. \\
& \left.\times G_{p, r, r}^{*}(t) G_{q, s, s}^{*}(t) G_{x, r, r}^{*}(t) G_{y, s, s}^{*}(t)\right]^{\frac{1}{6}}
\end{aligned}
$$

for all $\mathrm{x}, \mathrm{y}, \mathrm{p}, \mathrm{q}, \mathrm{r}, \mathrm{s} \in \mathrm{X}$ and $\mathrm{t}>0$. Then $\mathrm{T}$ has a unique fixed point in $\mathrm{X}$.

In Theorem 3.11, if we take $\varphi(t)=\lambda t(0<\lambda<1)$, then we have the following corollary.

Corollary 3.14. Let $\left(\mathrm{X}, \mathrm{G}^{*}, \Delta\right)$ be a complete PGM-space such that $\Delta$ is a $\mathrm{t}$-norm of $\mathrm{H}$-type. Let $\mathrm{T}: \mathrm{X} \times \mathrm{X} \rightarrow \mathrm{X}$ and $\mathrm{A}: \mathrm{X} \rightarrow \mathrm{X}$ be two mappings such that

$$
\begin{aligned}
G_{T(x, y), T(p, q), T(r, s)}^{*}(\lambda t) \geqslant & {\left[G_{A x, A p, A r}^{*}(t) G_{A y, A q, A s}^{*}(t) G_{A x, A p, A p}^{*}(t) G_{A y, A q, A q}^{*}(t)\right.} \\
& \left.\times G_{A p, A r, A r}^{*}(t) G_{A q, A s, A s}^{*}(t) G_{A x, A r, A r}^{*}(t) G_{A y, A s, A s}^{*}(t)\right]^{\frac{1}{6}}
\end{aligned}
$$

for all $x, y, p, q, r, s \in X$, where $\lambda \in(0,1), T(X \times X) \subset A(X), A$ is continuous and commutative with $T$. Then there exists a unique $\mathrm{u} \in \mathrm{X}$ such that $\mathrm{u}=\mathrm{Au}=\mathrm{T}(\mathrm{u}, \mathrm{u})$.

From the proof of Theorem 3.11, we can similarly prove the following result.

Theorem 3.15. Let $\left(\mathrm{X}, \mathrm{G}^{*}, \Delta\right)$ be a complete PGM-space such that $\Delta$ is a $\mathrm{t}$-norm of $\mathrm{H}$-type. Let $\varphi: \mathbb{R}^{+} \rightarrow \mathbb{R}^{+}$be a gauge function such that $\varphi^{-1}(\{0\})=\{0\}, \varphi(t)>t$ and $\lim _{n \rightarrow \infty} \varphi^{n}(t)=+\infty$ for any $\mathrm{t}>0$. Let $\mathrm{T}: \mathrm{X} \times \mathrm{X} \rightarrow \mathrm{X}$ and $\mathrm{A}: \mathrm{X} \rightarrow \mathrm{X}$ be two mappings such that

$$
\begin{aligned}
\mathrm{G}_{\mathrm{T}(\mathrm{x}, \mathrm{y}), \mathrm{T}(\mathrm{p}, \mathrm{q}), \mathrm{T}(\mathrm{r}, \mathrm{s})}^{*}(\mathrm{t}) \geqslant & {\left[\mathrm{G}_{A x, A p, A r}^{*}(\varphi(t)) \mathrm{G}_{A y, A q, A s}^{*}(\varphi(t))\right.} \\
& \times \mathrm{G}_{A x, A p, A p}^{*}(\varphi(t)) \mathrm{G}_{A y, A q, A q}^{*}(\varphi(t)) G_{A p, A r, A r}^{*}(\varphi(t)) \\
& \left.\times G_{A q, A s, A s}^{*}(\varphi(t)) G_{A x, A r, A r}^{*}(\varphi(t)) G_{A y, A s, A s}^{*}(\varphi(t))\right]^{\frac{1}{6}}
\end{aligned}
$$

for all $x, y, p, q, r, s \in X$, where $T(X \times X) \subset A(X), A$ is continuous and commutative with $T$. Then there exists a unique $\mathrm{u} \in \mathrm{X}$ such that $\mathrm{u}=\mathrm{A} \mathrm{u}=\mathrm{T}(\mathrm{u}, \mathrm{u})$.

Letting $A=I$ ( $\mathrm{I}$ is the identity mapping) in Theorem 3.15, we can obtain the following corollary. 
Corollary 3.16. Let $\left(\mathrm{X}, \mathrm{G}^{*}, \Delta\right)$ be a complete PGM-space such that $\Delta$ is a $\mathrm{t}$-norm of $\mathrm{H}$-type. Let $\varphi: \mathbb{R}^{+} \rightarrow \mathbb{R}^{+}$be a gauge function such that $\varphi^{-1}(\{0\})=\{0\}, \varphi(t)>t$ and $\lim _{n \rightarrow \infty} \varphi^{n}(t)=+\infty$ for any $\mathrm{t}>0$. Let $\mathrm{T}: \mathrm{X} \times \mathrm{X} \rightarrow \mathrm{X}$ be a mapping satisfying

$$
\begin{aligned}
G_{T(x, y), T(p, q), T(r, s)}^{*}(t) \geqslant & {\left[G_{x, p, r}^{*}(\varphi(t)) G_{y, q, s}^{*}(\varphi(t)) G_{x, p, p}^{*}(\varphi(t)) G_{y, q, q}^{*}(\varphi(t))\right.} \\
& \left.\times G_{p, r, r}^{*}(\varphi(t)) G_{q, s, s}^{*}(\varphi(t)) G_{x, r, r}^{*}(\varphi(t)) G_{y, s, s}^{*}(\varphi(t))\right]^{\frac{1}{6}}
\end{aligned}
$$

for all $\mathrm{x}, \mathrm{y}, \mathrm{p}, \mathrm{q}, \mathrm{r}, \mathrm{s} \in \mathrm{X}$ and $\mathrm{t}>0$. Then $\mathrm{T}$ has a unique fixed point in $\mathrm{X}$.

Theorem 3.17. Let $\left(\mathrm{X}, \mathrm{G}^{*}, \Delta\right)$ be a complete PGM-space such that $\Delta$ is a $\mathrm{t}$-norm of $\mathrm{H}$-type. Let $\varphi: \mathbb{R}^{+} \rightarrow \mathbb{R}^{+}$be a gauge function such that $\varphi^{-1}(\{0\})=\{0\}, \varphi(t)>t$ and $\lim _{n \rightarrow \infty} \varphi^{n}(t)=+\infty$ for any $t>0$. Let $\mathrm{T}: X \times X \rightarrow X$ and $\mathrm{A}: \mathrm{X} \rightarrow \mathrm{X}$ be two mappings such that

$$
\begin{aligned}
G_{T(x, y), T(p, q), T(r, s)}^{*}(t) \geqslant \min & \left\{G_{A x, A p, A r}^{*}(\varphi(t)), G_{A y, A q, A s}^{*}(\varphi(t)),\right. \\
& G_{A x, A p, A p}^{*}(\varphi(t)), G_{A y, A q, A q}^{*}(\varphi(t)), G_{A p, A r, A r}^{*}(\varphi(t)), \\
& \left.G_{A q, A s, A s}^{*}(\varphi(t)), G_{A x, A r, A r}^{*}(\varphi(t)), G_{A y, A s, A s}^{*}(\varphi(t))\right\}
\end{aligned}
$$

for all $x, y, p, q, r, s \in X$, where $T(X \times X) \subset A(X), A$ is continuous and commutative with $T$. Then there exists a unique $\mathrm{u} \in \mathrm{X}$ such that $\mathrm{u}=\mathrm{A} u=\mathrm{T}(\mathrm{u}, \mathrm{u})$.

Proof. Following the lines of the proof of Theorem 3.11, we can construct two sequences $\left\{x_{n}\right\}_{n=1}^{\infty}$ and $\left\{y_{n}\right\}_{n=1}^{\infty}$ in $X$ such that $A x_{n+1}=T\left(x_{n}, y_{n}\right)$ and $A y_{n+1}=T\left(y_{n}, x_{n}\right)$. By (3.23) we get (for $\left.t>0\right)$

$$
\begin{aligned}
G_{A x_{n}, A x_{n+1}, A x_{n+1}}^{*}(t) & =G_{T\left(x_{n-1}, y_{n-1}\right), T\left(x_{n}, y_{n}\right), T\left(x_{n}, y_{n}\right)}^{*}(t) \\
& \geqslant \min \left\{G_{A x_{n-1}, A x_{n}, A x_{n}}^{*}(\varphi(t)), G_{A y_{n-1}, A y_{n}, A y_{n}}^{*}(\varphi(t))\right\}
\end{aligned}
$$

and

$$
\begin{aligned}
G_{A y_{n}, A y_{n+1}, A y_{n+1}}^{*}(t) & =G_{T\left(y_{n-1}, x_{n-1}\right), T\left(y_{n}, x_{n}\right), T\left(y_{n}, x_{n}\right)}^{*}(t) \\
& \geqslant \min \left\{G_{A y_{n-1}, A y_{n}, A y_{n}}^{*}(\varphi(t)), G_{A x_{n-1}, A x_{n}, A x_{n}}^{*}(\varphi(t))\right\} .
\end{aligned}
$$

Denote $D_{n}(t)=\min \left\{G_{A x_{n-1}, A x_{n}, A x_{n}}^{*}(t), G_{A y_{n-1}, A y_{n}, A y_{n}}^{*}(t)\right\}$. By (3.24) and (3.25), it is easy to find that $D_{n+1}(t) \geqslant D_{n}(\varphi(t))$. This implies that

$$
D_{n+1}(t) \geqslant D_{n}(\varphi(t)) \geqslant D_{n-1}\left(\varphi^{2}(t)\right) \geqslant \cdots \geqslant D_{1}\left(\varphi^{n}(t)\right) .
$$

Since $\lim _{t \rightarrow+\infty} D_{1}(t)=\lim _{t \rightarrow+\infty} \min \left\{G_{A x_{0}, A x_{1}, A x_{1}}^{*}(t), G_{A y_{0}, A y_{1}, A y_{1}}^{*}(t)\right\}=1$ and $\lim _{n \rightarrow \infty} \varphi^{n}(t)=+\infty$ for each $t>0$, we have $\lim _{n \rightarrow \infty} D_{1}\left(\varphi^{n}(t)\right)=1$.

On the other hand, by using (3.24)-(3.26), we get

$$
G_{A x_{n}, A x_{n+1}, A x_{n+1}}^{*}(t) \geqslant D_{1}\left(\varphi^{n}(t)\right) \text { and } G_{A y_{n}, A y_{n+1}, A y_{n+1}}^{*}(t) \geqslant D_{1}\left(\varphi^{n}(t)\right) .
$$

Hence, we have

$$
\lim _{n \rightarrow \infty} G_{A x_{n}, A x_{n+1}, A x_{n+1}}^{*}(t)=1 \text { and } \lim _{n \rightarrow \infty} G_{A y_{n}, A y_{n+1}, A y_{n+1}}^{*}(t)=1
$$

These imply that

$$
\lim _{n \rightarrow \infty} D_{n}(t)=1 \quad \text { for all } t>0
$$

In the next step we show that, for any $k \in \mathbb{Z}^{+}$,

$$
G_{A x_{n}, A x_{n+k}, A x_{n+k}}^{*}(\varphi(t)) \geqslant \Delta^{k}\left(D_{n}(\varphi(t)-t)\right)
$$

and

$$
G_{A y_{n}, A y_{n+k}, A y_{n+k}}^{*}(\varphi(t)) \geqslant \Delta^{k}\left(D_{n}(\varphi(t)-t)\right)
$$


As $k=1$,

$$
\begin{aligned}
G_{A x_{n}, A x_{n+1}, A x_{n+1}}^{*}(\varphi(t)) & \geqslant G_{A x_{n}, A x_{n+1}, A x_{n+1}}^{*}(t) \\
& =G_{T\left(x_{n-1}, y_{n-1}\right), T\left(x_{n}, y_{n}\right), T\left(x_{n}, y_{n}\right)}^{*}(t) \\
& \geqslant \min \left\{G_{A x_{n-1}, A x_{n}, A x_{n}}^{*}(\varphi(t)), G_{A y_{n-1}, A y_{n}, A y_{n}}^{*}(\varphi(t))\right\} \\
& \geqslant \min \left\{G_{A x_{n-1}, A x_{n}, A x_{n}}^{*}(\varphi(t)-t), G_{A y_{n-1}, A y_{n}, A y_{n}}^{*}(\varphi(t)-t)\right\} \\
& =D_{n}(\varphi(t)-t) \\
& =\Delta\left(D_{n}(\varphi(t)-t), 1\right) \\
& \geqslant \Delta\left(D_{n}(\varphi(t)-t), D_{n}(\varphi(t)-t)\right) \\
& =\Delta^{1}\left(D_{n}(\varphi(t)-t)\right) .
\end{aligned}
$$

Similarly, we have

$$
G_{A y_{n}, A y_{n+1}, A y_{n+1}}^{*}(\varphi(t)) \geqslant \Delta^{1}\left(D_{n}(\varphi(t)-t)\right) .
$$

Thus (3.27) and (3.28) hold for $k=1$.

Assume that (3.27) and (3.28) hold for some fixed $k(k \geqslant 1)$. Since $\varphi(t)>t$, by (3.24) we have

$$
G_{A x_{n}, A x_{n+1}, A x_{n+1}}^{*}(t) \geqslant D_{n}(\varphi(t)) \geqslant D_{n}(t)
$$

for all $t>0$. By (3.23) and (3.27) we have

$$
\begin{aligned}
G_{A x_{n+1}, A_{x_{n+k}}, A_{x_{n+k+1}}(t)}^{*} & =G_{T\left(x_{n}, y_{n}\right), T\left(x_{n+k}, y_{n+k}\right), T\left(x_{n+k}, y_{n+k}\right)}^{*}(t) \\
& \geqslant \min \left\{G_{A x_{n}, A x_{n+k}, A x_{n+k}}^{*}(\varphi(t)), G_{A y_{n}, A y_{n+k}, A y_{n+k}}^{*}(\varphi(t))\right\} \\
& \geqslant \Delta^{k}\left(D_{n}(\varphi(t)-t)\right) .
\end{aligned}
$$

Thus, by (PGM-4), (3.29), (3.30), and the monotonicity of $\Delta$, we have

$$
\begin{aligned}
\mathrm{G}_{A x_{n}, A x_{n+k+1}, A x_{n+k+1}}^{*}(\varphi(t)) & =G_{A x_{n}, A x_{n+k+1}, A x_{n+k+1}}^{*}(\varphi(t)-t+t) \\
& \geqslant \Delta\left(G_{A x_{n}, A x_{n+1}, A x_{n+1}}^{*}(\varphi(t)-t), G_{A x_{n+1}, A x_{n+k+1}, A x_{n+k+1}}^{*}(t)\right) \\
& \geqslant \Delta\left(D_{n}(\varphi(t)-t), \Delta^{k}\left(D_{n}(\varphi(t)-t)\right)\right) \\
& =\Delta^{k+1}\left(D_{n}(\varphi(t)-t)\right) .
\end{aligned}
$$

Similarly, we have $G_{A y_{n}, A y_{n+k+1}, A y_{n+k+1}}^{*}(\varphi(t)) \geqslant \Delta^{k+1}\left(D_{n}(\varphi(t)-t)\right)$. Hence, by induction, (3.27) and (3.28) hold for all $k \in \mathbb{Z}^{+}$.

By the same method as in Theorem 3.11, we can obtain that $\left\{A x_{n}\right\}$ and $\left\{A y_{n}\right\}$ are all Cauchy sequences. Since $X$ is complete, there exist $u, v \in X$ such that $\lim _{n \rightarrow \infty} A x_{n}=u$ and $\lim _{n \rightarrow \infty} A y_{n}=v$. From the continuity of $A$ it follows that

$$
\lim _{n \rightarrow \infty} A A x_{n}=A u \text { and } \lim _{n \rightarrow \infty} A A y_{n}=A v .
$$

From (3.23) and the commutativity of A with $\mathrm{T}$ it follows that

$$
\begin{aligned}
\mathrm{G}_{A A x_{n+1}, T(u, v), T(u, v)}^{*}(t) & =\mathrm{G}_{A \mathrm{~T}\left(x_{n}, y_{n}\right), T(u, v), T(u, v)}^{*}(t) \\
& =\mathrm{G}_{\mathrm{T}\left(A x_{n}, A y_{n}\right), T(u, v), T(u, v)}^{*}(t) \\
& \geqslant \min \left\{G_{A A x_{n}, A u, A u}^{*}(\varphi(t)), G_{A A y_{n}, A v, A v}^{*}(\varphi(t))\right\} .
\end{aligned}
$$

Now on making $n \rightarrow \infty$ in (3.31), we have $\lim _{n \rightarrow \infty} A A x_{n}=T(u, v)$. Hence, $A u=T(u, v)$. In the same manner we can prove that $A v=T(v, u)$. 
Next, we shall show that $A u=v$ and $A v=u$. In fact, by (3.23) we have

$$
\begin{aligned}
\mathrm{G}_{A u, A y_{n}, A y_{n}}^{*}(t) & =\mathrm{G}_{\mathrm{T}(u, v), T\left(y_{n-1}, x_{n-1}\right), T\left(y_{n-1}, x_{n-1}\right)}^{*}(t) \\
& \geqslant \min \left\{G_{A u, A y_{n-1}, A y_{n-1}}^{*}(\varphi(t)), G_{A v, A x_{n-1}, A x_{n-1}}^{*}(\varphi(t))\right\}
\end{aligned}
$$

and

$$
\begin{aligned}
& \mathrm{G}_{A u, A x_{n}, A x_{n}}^{*}(t)=G_{T}^{*}(u, v), T\left(x_{n-1}, y_{n-1}\right), T\left(x_{n-1}, y_{n-1}\right)(t) \\
& \geqslant \min \left\{G_{A u, A x_{n-1}, A x_{n-1}}^{*}(\varphi(t)), G_{A v, A y_{n-1}, A y_{n-1}}^{*}(\varphi(t))\right\} \text {. }
\end{aligned}
$$

Denote $E_{n}(t)=\min \left\{G_{A u, A y_{n}, A y_{n}}^{*}(t), G_{A v, A x_{n}, A x_{n}}^{*}(t)\right\}$. From (3.32) and (3.33), it is easy to find that

$$
E_{n}(t) \geqslant E_{n-1}(\varphi(t)) \geqslant \cdots \geqslant E_{0}\left(\varphi^{n}(t)\right) .
$$

Since $\lim _{n \rightarrow \infty} \varphi_{n}(t)=+\infty$, we have

$$
\mathrm{E}_{0}\left(\varphi^{\mathrm{n}}(\mathrm{t})\right)=\min \left\{\mathrm{G}_{A \mathrm{u}, A \mathrm{y}_{0}, A y_{0}}^{*}\left(\varphi^{\mathrm{n}}(\mathrm{t})\right), \mathrm{G}_{A \nu, A x_{0}, A x_{0}}^{*}\left(\varphi^{\mathrm{n}}(\mathrm{t})\right)\right\} \rightarrow 1
$$

as $n \rightarrow \infty$. This shows that $E_{n}(t) \rightarrow 1$ as $n \rightarrow \infty$, and so

$$
\lim _{n \rightarrow \infty} A y_{n}=A u \text { and } \lim _{n \rightarrow \infty} A x_{n}=A v .
$$

Hence, $A u=v$ and $A v=u$.

Finally, we shall prove that $u=v$. From (3.23) we get

$$
\begin{aligned}
\mathrm{G}_{u, v, v}^{*}(\mathrm{t})=\mathrm{G}_{A v, A u, A u}^{*}(\mathrm{t}) & =\mathrm{G}_{\mathrm{T}(v, u), T(u, v), T(u, v)}^{*}(\mathrm{t}) \\
& \geqslant \min \left\{\mathrm{G}_{A v, A u, A u}^{*}(\varphi(t)), \mathrm{G}_{A u, A v, A v}^{*}(\varphi(t))\right\} \\
& =\min \left\{\mathrm{G}_{u, v, v}^{*}(\varphi(t)), \mathrm{G}_{v, u, u}^{*}(\varphi(t))\right\}
\end{aligned}
$$

and

$$
\begin{aligned}
\mathrm{G}_{v, u, u}^{*}(t)=\mathrm{G}_{A u, A v, A v}^{*}(t) & =\mathrm{G}_{\mathrm{T}(\mathrm{u}, v), \mathrm{T}(v, \mathrm{u}), \mathrm{T}(v, u)}^{*}(\mathrm{t}) \\
& \geqslant \min \left\{\mathrm{G}_{A u, A v, A v}^{*}(\varphi(t)), \mathrm{G}_{A v, A u, A u}^{*}(\varphi(t))\right\} \\
& =\min \left\{\mathrm{G}_{v, u, u}^{*}(\varphi(t)), \mathrm{G}_{u, v, v}^{*}(\varphi(t))\right\} .
\end{aligned}
$$

Suppose that $F(t)=\min \left\{G_{u, v, v}^{*}(t), G_{v, u, u}^{*}(t)\right\}$. From (3.34) and (3.35), we obtain that $F(t) \geqslant F\left(\varphi^{n}(t)\right)$. Letting $n \rightarrow \infty$, we have $F(t)=1$, i.e., $u=v$. Since the uniqueness of $u$ follows from (3.23), the proof of Theorem 3.17 is completed.

Remark 3.18. Theorem 3.17 generalizes and extends the corresponding result in Xiao et al. [22].

Letting $A=I$ (I is the identity mapping) in Theorem 3.17, we can obtain the following corollary.

Corollary 3.19. Let $\left(\mathrm{X}, \mathrm{G}^{*}, \Delta\right)$ be a complete PGM-space such that $\Delta$ is a $\mathrm{t}$-norm of $\mathrm{H}$-type. Let $\varphi: \mathbb{R}^{+} \rightarrow \mathbb{R}^{+}$be a gauge function such that $\varphi^{-1}(\{0\})=\{0\}, \varphi(t)>t$ and $\lim _{n \rightarrow \infty} \varphi^{n}(t)=+\infty$ for any $\mathrm{t}>0$. Let $\mathrm{T}: \mathrm{X} \times \mathrm{X} \rightarrow \mathrm{X}$ be a mapping such that

$$
\begin{array}{r}
G_{T(x, y), T(p, q), T(r, s)}^{*}(t) \geqslant \min \left\{G_{x, p, r}^{*}(\varphi(t)), G_{y, q, s}^{*}(\varphi(t)), G_{x, p, p}^{*}(\varphi(t)), G_{y, q, q}^{*}(\varphi(t)),\right. \\
\left.G_{p, r, r}^{*}(\varphi(t)), G_{q, s, s}^{*}(\varphi(t)), G_{x, r, r}^{*}(\varphi(t)), G_{y, s, s}^{*}(\varphi(t))\right\}
\end{array}
$$

for all $\mathrm{x}, \mathrm{y}, \mathrm{p}, \mathrm{q}, \mathrm{r}, \mathrm{s} \in \mathrm{X}$ and $\mathrm{t}>0$. Then $\mathrm{T}$ has a unique fixed point in $\mathrm{X}$.

By the same method as in Theorem 3.17, we can obtain the following theorem. 
Theorem 3.20. Let $\left(\mathrm{X}, \mathrm{G}^{*}, \Delta\right)$ be a complete PGM-space such that $\Delta$ is a $\mathrm{t}$-norm of $\mathrm{H}$-type. Let $\varphi: \mathbb{R}^{+} \rightarrow \mathbb{R}^{+}$be a gauge function such that $\varphi^{-1}(\{0\})=\{0\}, \varphi(t)<\mathrm{t}$ and $\lim _{\mathrm{n} \rightarrow \infty} \varphi^{\mathrm{n}}(\mathrm{t})=0$ for any $\mathrm{t}>0$. Let $\mathrm{T}: \mathrm{X} \times \mathrm{X} \rightarrow \mathrm{X}$ and $\mathrm{A}: \mathrm{X} \rightarrow \mathrm{X}$ be two mappings such that

$$
\begin{array}{r}
G_{T(x, y), T(p, q), T(r, s)}^{*}(\varphi(t)) \geqslant \min \left\{G_{A x, A p, A r}^{*}(t), G_{A y, A q, A s}^{*}(t), G_{A x, A p, A p}^{*}(t), G_{A y, A q, A q}^{*}(t),\right. \\
\left.G_{A p, A r, A r}^{*}(t), G_{A q, A s, A s}^{*}(t), G_{A x, A r, A r}^{*}(t), G_{A y, A s, A s}^{*}(t)\right\}
\end{array}
$$

for all $x, y, p, q, r, s \in X$, where $T(X \times X) \subset A(X), A$ is continuous and commutative with $T$. Then there exists a unique $\mathrm{u} \in \mathrm{X}$ such that $\mathrm{u}=\mathrm{Au}=\mathrm{T}(\mathrm{u}, \mathrm{u})$.

In Theorem 3.20, if we take $A=I$ ( $\mathrm{I}$ is the identity mapping), then we have the following corollary.

Corollary 3.21. Let $\left(\mathrm{X}, \mathrm{G}^{*}, \Delta\right)$ be a complete PGM-space such that $\Delta$ is a $\mathrm{t}$-norm of $\mathrm{H}$-type. Let $\varphi: \mathbb{R}^{+} \rightarrow \mathbb{R}^{+}$be a gauge function such that $\varphi^{-1}(\{0\})=\{0\}, \varphi(t)<\mathrm{t}$ and $\lim _{\mathrm{n} \rightarrow \infty} \varphi^{\mathrm{n}}(\mathrm{t})=0$ for any $\mathrm{t}>0$. Let $\mathrm{T}: \mathrm{X} \times \mathrm{X} \rightarrow \mathrm{X}$ be a mapping satisfying

$$
\begin{array}{r}
G_{T(x, y), T(p, q), T(r, s)}^{*}(\varphi(t)) \geqslant \min \left\{G_{x, p, r}^{*}(t), G_{y, q, s}^{*}(t), G_{x, p, p}^{*}(t), G_{y, q, q}^{*}(t),\right. \\
\left.G_{p, r, r}^{*}(t), G_{q, s, s}^{*}(t), G_{x, r, r}^{*}(t), G_{y, s, s}^{*}(t)\right\}
\end{array}
$$

for all $\mathrm{x}, \mathrm{y}, \mathrm{p}, \mathrm{q}, \mathrm{r}, \mathrm{s} \in \mathrm{X}$ and $\mathrm{t}>0$. Then $\mathrm{T}$ has a unique fixed point in $\mathrm{X}$.

In Theorem 3.20, if we take $\varphi(t)=\lambda t(0<\lambda<1)$, then we have the following corollary.

Corollary 3.22. Let $\left(\mathrm{X}, \mathrm{G}^{*}, \Delta\right)$ be a complete PGM-space such that $\Delta$ is a $\mathrm{t}$-norm of $\mathrm{H}$-type. Let $\mathrm{T}: \mathrm{X} \times \mathrm{X} \rightarrow \mathrm{X}$ and $\mathrm{A}: \mathrm{X} \rightarrow \mathrm{X}$ be two mappings such that

$$
\begin{array}{r}
G_{T(x, y), T(p, q), T(r, s)}^{*}(\lambda t) \geqslant \min \left\{G_{A x, A p, A r}^{*}(t), G_{A y, A q, A s}^{*}(t), G_{A x, A p, A p}^{*}(t), G_{A y, A q, A q}^{*}(t),\right. \\
\left.G_{A p, A r, A r}^{*}(t), G_{A q, A s, A s}^{*}(t), G_{A x, A r, A r}^{*}(t), G_{A y, A s, A s}^{*}(t)\right\}
\end{array}
$$

for all $x, y, p, q, r, s \in X$, where $\lambda \in(0,1), T(X \times X) \subset A(X), A$ is continuous and commutative with $T$. Then there exists a unique $\mathrm{u} \in \mathrm{X}$ such that $\mathrm{u}=\mathrm{Au}=\mathrm{T}(\mathrm{u}, \mathrm{u})$.

Now, we give the common tripled fixed point results under probabilistic $\varphi$-contractive conditions in PGM-space.

Theorem 3.23. Let $\left(\mathrm{X}, \mathrm{G}^{*}, \Delta\right)$ be a complete PGM-space such that $\Delta$ is a $\mathrm{t}$-norm of $\mathrm{H}$-type. Let $\varphi: \mathbb{R}^{+} \rightarrow \mathbb{R}^{+}$be a gauge function such that $\varphi^{-1}(\{0\})=\{0\}, \varphi(t)<\mathrm{t}$ and $\lim _{\mathrm{n} \rightarrow \infty} \varphi^{\mathrm{n}}(\mathrm{t})=0$ for any $\mathrm{t}>0$. Let $\mathrm{T}: \mathrm{X} \times \mathrm{X} \times \mathrm{X} \rightarrow \mathrm{X}$ and $\mathrm{A}: \mathrm{X} \rightarrow \mathrm{X}$ be two mappings such that

$$
\begin{aligned}
\mathrm{G}_{\mathrm{T}(x, y, \zeta), \mathrm{T}(\mathrm{p}, \mathrm{q}, \eta), \mathrm{T}(\mathrm{r}, \mathrm{s}, \theta)}^{*}(\varphi(t)) \geqslant & {\left[\mathrm{G}_{A x, A p, A r}^{*}(t) G_{A y, A q, A s}^{*}(t) G_{A \zeta, A \eta, A \theta}^{*}(t)\right.} \\
& \times G_{A x, A p, A p}^{*}(t) G_{A y, A q, A q}^{*}(t) G_{A \zeta, A \eta, A \eta}^{*}(t) \\
& \times G_{A p, A r, A r}^{*}(t) G_{A q, A s, A s}^{*}(t) G_{A \eta, A \theta, A \theta}^{*}(t) \\
& \left.\times G_{A x, A r, A r}^{*}(t) G_{A y, A s, A s}^{*}(t) G_{A \zeta, A \theta, A \theta}^{*}(t)\right]^{\frac{1}{9}}
\end{aligned}
$$

for all $x, y, p, q, r, s, \zeta, \eta, \theta \in X$, where $T(X \times X \times X) \subset A(X), A$ is continuous and commutative with $T$. Then there exists a unique $\mathrm{u} \in \mathrm{X}$ such that $\mathrm{u}=\mathrm{Au}=\mathrm{T}(\mathrm{u}, \mathrm{u}, \mathrm{u})$.

Proof. Let $x_{0}, y_{0}, \zeta_{0}$ be any given points of $X$. Since $T(X \times X \times X) \subset A(X)$, we can choose $x_{1}, y_{1}, \zeta_{1} \in$ $X$ such that $A x_{1}=T\left(x_{0}, y_{0}, \zeta_{0}\right), A y_{1}=T\left(y_{0}, x_{0}, \zeta_{0}\right)$, and $A \zeta_{1}=T\left(\zeta_{0}, x_{0}, y_{0}\right)$. Again from $T(X \times X \times$ $X) \subset A(X)$, we can choose $x_{2}, y_{2}, \zeta_{2} \in X$ such that $A x_{2}=T\left(x_{1}, y_{1}, \zeta_{1}\right), A y_{2}=T\left(y_{1}, x_{1}, \zeta_{1}\right)$, and $A \zeta_{2}=$ $T\left(\zeta_{1}, x_{1}, y_{1}\right)$. Continuing this process, we can construct sequences $\left\{x_{n}\right\},\left\{y_{n}\right\}$, and $\left\{\zeta_{n}\right\}$ in $X$ such that $A x_{n+1}=T\left(x_{n}, y_{n}, \zeta_{n}\right), A y_{n+1}=T\left(y_{n}, x_{n}, \zeta_{n}\right)$, and $A \zeta_{n+1}=T\left(\zeta_{n}, x_{n}, y_{n}\right)$ for all $n \in \mathbb{N}$. 
By the same methods as in Theorem 3.11, we can prove that $\left\{A x_{n}\right\},\left\{A y_{n}\right\}$, and $\left\{A \zeta_{n}\right\}$ are all Cauchy sequences. Since $X$ is complete, there exist $u, v, w \in X$ such that $\lim _{n \rightarrow \infty} A x_{n}=u, \lim _{n \rightarrow \infty} A y_{n}=v$, and $\lim _{n \rightarrow \infty} A \zeta_{n}=w$. From the continuity of $A$ it follows that

$$
\lim _{n \rightarrow \infty} A A x_{n}=A u, \quad \lim _{n \rightarrow \infty} A A y_{n}=A v, \text { and } \lim _{n \rightarrow \infty} A A \zeta_{n}=A w .
$$

From (3.36) and the commutativity of A with T, we obtain that

$$
\begin{aligned}
\mathrm{G}_{A A x_{n+1}, T(u, v, w), T(u, v, w)}^{*}(\varphi(t)) & =G_{A T\left(x_{n}, y_{n}, \zeta_{n}\right), T(u, v, w), T(u, v, w)}^{*}(\varphi(t)) \\
& =G_{T\left(A x_{n}, A y_{n}, A \zeta_{n}\right), T(u, v, w), T(u, v, w)}^{*}(\varphi(t)) \\
& \geqslant\left[G_{A A x_{n}, A u, A u}^{*}(t) G_{A A y_{n}, A v, A v}^{*}(t) G_{A A \zeta_{n}, A w, A w}^{*}(t)\right]^{\frac{1}{3}} .
\end{aligned}
$$

Now on making $n \rightarrow \infty$ in (3.37), we get $\lim _{n \rightarrow \infty} A A x_{n}=T(u, v, w)$. Hence, $A u=T(u, v, w)$. In the same manner we can prove that $A v=\mathrm{T}(v, \mathrm{u}, w)$ and $A w=\mathrm{T}(w, u, v)$.

Next, we shall show that $A u=v, A v=u$, and $A w=w$. In fact, by (3.36) we have

$$
\begin{aligned}
\mathrm{G}_{A u, A y_{n}, A y_{n}}^{*}(\varphi(t)) & =G_{T}^{*}(u, v, w), T\left(y_{n-1}, x_{n-1}, \zeta_{n-1}\right), T\left(y_{n-1}, x_{n-1}, \zeta_{n-1}\right) \\
& \geqslant\left[G_{A u, A y_{n-1}, A y_{n-1}}^{*}(t) G_{A v, A x_{n-1}, A x_{n-1}}^{*}(t) G_{A w, A \zeta_{n-1}, A \zeta_{n-1}}^{*}(t)\right]^{\frac{1}{3}}, \\
G_{A v, A x_{n}, A x_{n}}^{*}(\varphi(t)) & =G_{T(v, u, w), T\left(x_{n-1}, y_{n-1}, \zeta_{n-1}\right), T\left(x_{n-1}, y_{n-1}, \zeta_{n-1}\right)}^{*}(\varphi(t)) \\
& \geqslant\left[G_{A v, A x_{n-1}, A x_{n-1}}^{*}(t) G_{A u, A y_{n-1}, A y_{n-1}}^{*}(t) G_{A w, A \zeta_{n-1}, A \zeta_{n-1}}^{*}(t)\right]^{\frac{1}{3}},
\end{aligned}
$$

and

$$
\begin{aligned}
\mathrm{G}_{A w, A \zeta_{n}, A \zeta_{n}}^{*}(\varphi(t)) & =\mathrm{G}_{\mathrm{T}(w, u, v), T\left(\zeta_{n-1}, x_{n-1}, y_{n-1}\right), T\left(\zeta_{n-1}, x_{n-1}, y_{n-1}\right)}^{*}(\varphi(t)) \\
& \geqslant\left[G_{A w, A \zeta_{n-1}, A \zeta_{n-1}}^{*}(t) G_{A u, A x_{n-1}, A x_{n-1}}^{*}(t) G_{A v, A y_{n-1}, A y_{n-1}}^{*}(t)\right]^{\frac{1}{3}} .
\end{aligned}
$$

Denote $H_{n}(t)=G_{A u, A y_{n}, A y_{n}}^{*}(t) G_{A v, A x_{n}, A x_{n}}^{*}(t) G_{A w, A \zeta_{n}, A \zeta_{n}}^{*}(t)$. From (3.38), (3.39), and (3.40), we find that

$$
\mathrm{H}_{\mathrm{n}}\left(\varphi^{\mathrm{n}}(\mathrm{t})\right) \geqslant \mathrm{H}_{\mathrm{n}-1}\left(\varphi^{\mathrm{n}-1}(\mathrm{t})\right) \geqslant \cdots \geqslant \mathrm{H}_{0}(\mathrm{t}) .
$$

Since $\lim _{n \rightarrow \infty} \varphi_{n}(t)=0$, from Lemma 2.10, we have

$$
H_{n}(t)=G_{A u, A y_{n}, A y_{n}}^{*}(t) G_{A v, A x_{n}, A x_{n}}^{*}(t) G_{A w, A \zeta_{n}, A \zeta_{n}}^{*}(t) \rightarrow 1
$$

as $n \rightarrow \infty$. This shows that

$$
\lim _{n \rightarrow \infty} A y_{n}=A u, \lim _{n \rightarrow \infty} A x_{n}=A v, \lim _{n \rightarrow \infty} A \zeta_{n}=A w .
$$

Hence, $A u=v, A v=u, A w=w$.

Finally, we shall prove that $u=v$ and $u=w$. From (3.36) we get

$$
\begin{aligned}
& \mathrm{G}_{u, v, v}^{*}(\varphi(\mathrm{t}))=\mathrm{G}_{\mathrm{A} v, \mathrm{Au}, \mathrm{Au}}^{*}(\varphi(\mathrm{t}))=\mathrm{G}_{\mathrm{T}(v, u, w), \mathrm{T}(\mathrm{u}, v, w), \mathrm{T}(\mathrm{u}, v, w)}^{*}(\varphi(\mathrm{t})) \\
& \geqslant\left[G_{A v, A u, A u}^{*}(t) G_{A u, A v, A v}^{*}(t) G_{A w, A w, A w}^{*}(t)\right]^{\frac{1}{3}}=\left[G_{u, v, v}^{*}(t) G_{v, u, u}^{*}(t)\right]^{\frac{1}{3}},
\end{aligned}
$$

and

$$
\begin{aligned}
\mathrm{G}_{v, u, u}^{*}(\varphi(t)) & =\mathrm{G}_{A u, A v, A v}^{*}(\varphi(t))=\mathrm{G}_{\mathrm{T}(\mathrm{u}, v, w), \mathrm{T}(v, \mathrm{u}, w), \mathrm{T}(v, \mathrm{u}, w)}^{*}(\varphi(t)) \\
& \geqslant\left[\mathrm{G}_{\mathrm{Au}, \mathrm{A} v, \mathrm{~A} v}^{*}(\mathrm{t}) \mathrm{G}_{\mathrm{A} v, \mathrm{Au}, \mathrm{Au}}^{*}(\mathrm{t}) \mathrm{G}_{\mathrm{A} w, \mathrm{~A} w, \mathrm{~A} w}^{*}(\mathrm{t})\right]^{\frac{1}{3}}=\left[\mathrm{G}_{v, \mathrm{u}, \mathrm{u}}^{*}(\mathrm{t}) \mathrm{G}_{\mathrm{u}, v, v}^{*}(\mathrm{t})\right]^{\frac{1}{3}} .
\end{aligned}
$$

Suppose that $I(t)=G_{u, v, v}^{*}(t) G_{v, u, u}^{*}(t)$. From (3.41) and (3.42), we obtain that $I(\varphi(t)) \geqslant[I(t)]^{\frac{2}{3}}$. Hence, we have $I\left(\varphi^{n}(t)\right) \geqslant[I(t)]^{\left.]_{3}^{2}\right)^{n}}$. From Lemma 2.10, we get $I(t)=1$ for all $t>0$, i.e., $u=v$. Similarly, we can prove that $u=w$. Hence, there exists $u \in X$, such that $u=A u=T(u, u, u)$. Since the uniqueness of $u$ follows from (3.36), the proof of Theorem 3.23 is completed. 
In Theorem 3.23, if we take $A=I$ ( $\mathrm{I}$ is the identity mapping), then we have the following corollary.

Corollary 3.24. Let $\left(\mathrm{X}, \mathrm{G}^{*}, \Delta\right)$ be a complete PGM-space such that $\Delta$ is a $\mathrm{t}$-norm of $\mathrm{H}$-type. Let $\varphi: \mathbb{R}^{+} \rightarrow \mathbb{R}^{+}$be a gauge function such that $\varphi^{-1}(\{0\})=\{0\}, \varphi(t)<\mathrm{t}$ and $\lim _{\mathrm{n} \rightarrow \infty} \varphi^{\mathrm{n}}(\mathrm{t})=0$ for any $\mathrm{t}>0$. Let $\mathrm{T}: \mathrm{X} \times \mathrm{X} \times \mathrm{X} \rightarrow \mathrm{X}$ be a mapping satisfying

$$
\begin{aligned}
& \mathrm{G}_{\mathrm{T}(\mathrm{x}, \mathrm{y}, \zeta), T(p, q, \eta), T(r, s, \theta)}^{*}(\varphi(t)) \geqslant\left[\mathrm{G}_{x, p, r}^{*}(t) \mathrm{G}_{y, q, s}^{*}(t) \mathrm{G}_{\zeta, \eta, \theta}^{*}(t) \mathrm{G}_{x, p, p}^{*}(t) G_{y, q, q}^{*}(t)\right. \\
& \left.\times G_{\zeta, \eta, \eta}^{*}(t) G_{p, r, r}^{*}(t) G_{q, s, s}^{*}(t) G_{\eta, \theta, \theta}^{*}(t) G_{x, r, r}^{*}(t) G_{y, s, s}^{*}(t) G_{\zeta, \theta, \theta}^{*}(t)\right]^{\frac{1}{9}}
\end{aligned}
$$

for all $\mathrm{x}, \mathrm{y}, \mathrm{p}, \mathrm{q}, \mathrm{r}, \mathrm{s}, \zeta, \eta, \theta \in \mathrm{X}$ and $\mathrm{t}>0$. Then $\mathrm{T}$ has a unique fixed point in $\mathrm{X}$.

In Theorem 3.23, if we take $\varphi(t)=\lambda t(0<\lambda<1)$, then we have the following corollary.

Corollary 3.25. Let $\left(\mathrm{X}, \mathrm{G}^{*}, \Delta\right)$ be a complete PGM-space such that $\Delta$ is a $\mathrm{t}$-norm of $\mathrm{H}$-type. Let $\mathrm{T}: \mathrm{X} \times \mathrm{X} \times \mathrm{X} \rightarrow \mathrm{X}$ and $\mathrm{A}: \mathrm{X} \rightarrow \mathrm{X}$ be two mappings such that

$$
\begin{aligned}
\mathrm{G}_{\mathrm{T}(\mathrm{x}, \mathrm{y}, \zeta), T(\mathrm{p}, \mathrm{q}, \eta), T(\mathrm{r}, \mathrm{s}, \theta)}^{*}(\lambda t) \geqslant & {\left[\mathrm{G}_{A x, A p, A r}^{*}(t) G_{A y, A q, A s}^{*}(t) G_{A \zeta, A \eta, A \theta}^{*}(t)\right.} \\
& \times G_{A x, A p, A p}^{*}(t) G_{A y, A q, A q}^{*}(t) G_{A \zeta, A \eta, A \eta}^{*}(t) \\
& \times G_{A p, A r, A r}^{*}(t) G_{A q, A s, A s}^{*}(t) G_{A \eta, A \theta, A \theta}^{*}(t) \\
& \left.\times G_{A x, A r, A r}^{*}(t) G_{A y, A s, A s}^{*}(t) G_{A \zeta, A \theta, A \theta}^{*}(t)\right]^{\frac{1}{9}}
\end{aligned}
$$

for all $x, y, p, q, r, s, \zeta, \eta, \theta \in X$, where $\lambda \in(0,1), T(X \times X \times X) \subset A(X), A$ is continuous and commutative with $\mathrm{T}$. Then there exists a unique $\mathrm{u} \in \mathrm{X}$ such that $\mathrm{u}=\mathrm{Au}=\mathrm{T}(\mathrm{u}, \mathrm{u}, \mathrm{u})$.

In a similar way, we can prove the following result.

Theorem 3.26. Let $\left(\mathrm{X}, \mathrm{G}^{*}, \Delta\right)$ be a complete PGM-space such that $\Delta$ is a $\mathrm{t}$-norm of $\mathrm{H}$-type. Let $\varphi: \mathbb{R}^{+} \rightarrow \mathbb{R}^{+}$ be a gauge function such that $\varphi^{-1}(\{0\})=\{0\}, \varphi(t)>\mathrm{t}$ and $\lim _{\mathrm{n} \rightarrow \infty} \varphi^{\mathrm{n}}(\mathrm{t})=+\infty$ for any $\mathrm{t}>0$. Let $\mathrm{T}$ : $\mathrm{X} \times \mathrm{X} \times \mathrm{X} \rightarrow \mathrm{X}$ and $\mathrm{A}: \mathrm{X} \rightarrow \mathrm{X}$ be two mappings such that

$$
\begin{aligned}
\mathrm{G}_{\mathrm{T}(x, y, \zeta), T(p, q, \eta), T(r, s, \theta)}^{*}(t) \geqslant & {\left[\mathrm{G}_{A x, A p, A r}^{*}(\varphi(t)) \mathrm{G}_{A y, A q, A s}^{*}(\varphi(t)) G_{A \zeta, A \eta, A \theta}^{*}(\varphi(t))\right.} \\
& \times G_{A x, A p, A p}^{*}(\varphi(t)) G_{A y, A q, A q}^{*}(\varphi(t)) G_{A \zeta, A \eta, A \eta}^{*}(\varphi(t)) \\
& \times G_{A p, A r, A r}^{*}(\varphi(t)) G_{A q, A s, A s}^{*}(\varphi(t)) G_{A \eta, A \theta, A \theta}^{*}(\varphi(t)) \\
& \left.\times G_{A x, A r, A r}^{*}(\varphi(t)) G_{A y, A s, A s}^{*}(\varphi(t)) G_{A \zeta, A \theta, A \theta}^{*}(\varphi(t))\right]^{\frac{1}{9}}
\end{aligned}
$$

for all $x, y, p, q, r, s, \zeta, \eta, \theta \in X$, where $T(X \times X \times X) \subset A(X), A$ is continuous and commutative with $T$. Then there exists a unique $\mathrm{u} \in \mathrm{X}$ such that $\mathrm{u}=\mathrm{Au}=\mathrm{T}(\mathrm{u}, \mathrm{u}, \mathrm{u})$.

Letting $A=I$ (I is the identity mapping) in Theorem 3.26, we can obtain the following corollary.

Corollary 3.27. Let $\left(\mathrm{X}, \mathrm{G}^{*}, \Delta\right)$ be a complete PGM-space such that $\Delta$ is a $\mathrm{t}$-norm of $\mathrm{H}$-type. Let $\varphi: \mathbb{R}^{+} \rightarrow \mathbb{R}^{+}$ be a gauge function such that $\varphi^{-1}(\{0\})=\{0\}, \varphi(t)>\mathrm{t}$ and $\lim _{\mathrm{n} \rightarrow \infty} \varphi^{\mathrm{n}}(\mathrm{t})=+\infty$ for any $\mathrm{t}>0$. Let $\mathrm{T}$ : $\mathrm{X} \times \mathrm{X} \times \mathrm{X} \rightarrow \mathrm{X}$ be a mapping satisfying

$$
\begin{aligned}
\mathrm{G}_{\mathrm{T}(x, y, \zeta), T(p, q, \eta), T(r, s, \theta)}^{*}(t) \geqslant & {\left[\mathrm{G}_{x, p, r}^{*}(\varphi(t)) G_{y, q, s}^{*}(\varphi(t)) G_{\zeta, \eta, \theta}^{*}(\varphi(t))\right.} \\
& \times G_{x, p, p}^{*}(\varphi(t)) G_{y, q, q}^{*}(\varphi(t)) G_{\zeta, \eta, \eta}^{*}(\varphi(t)) \\
& \times G_{p, r, r}^{*}(\varphi(t)) G_{q, s, s}^{*}(\varphi(t)) G_{\eta, \theta, \theta}^{*}(\varphi(t)) \\
& \left.\times G_{x, r, r}^{*}(\varphi(t)) G_{y, s, s}^{*}(\varphi(t)) G_{\zeta, \theta, \theta}^{*}(\varphi(t))\right]^{\frac{1}{9}}
\end{aligned}
$$

for all $\mathrm{x}, \mathrm{y}, \mathrm{p}, \mathrm{q}, \mathrm{r}, \mathrm{s}, \zeta, \eta, \theta \in \mathrm{X}$ and $\mathrm{t}>0$. Then $\mathrm{T}$ has a unique fixed point in $\mathrm{X}$. 
From the proof of Theorem 3.23, we can similarly prove the following result.

Theorem 3.28. Let $\left(\mathrm{X}, \mathrm{G}^{*}, \Delta\right)$ be a complete PGM-space such that $\Delta$ is a $\mathrm{t}$-norm of $\mathrm{H}$-type. Let $\varphi: \mathbb{R}^{+} \rightarrow \mathbb{R}^{+}$be a gauge function such that $\varphi^{-1}(\{0\})=\{0\}, \varphi(t)>\mathrm{t}$ and $\lim _{\mathrm{n} \rightarrow \infty} \varphi^{\mathrm{n}}(\mathrm{t})=+\infty$ for any $\mathrm{t}>0$. Let $\mathrm{T}: \mathrm{X} \times \mathrm{X} \times \mathrm{X} \rightarrow$ $\mathrm{X}$ and $\mathrm{A}: \mathrm{X} \rightarrow \mathrm{X}$ be two mappings such that

$$
\begin{aligned}
& \mathrm{G}_{\mathrm{T}(\mathrm{x}, \mathrm{y}, \zeta), \mathrm{T}(\mathrm{p}, \mathrm{q}, \eta), \mathrm{T}(\mathrm{r}, \mathrm{s}, \theta)}^{*}(\mathrm{t}) \geqslant \min \left\{\mathrm{G}_{A x, A p, A r}^{*}(\varphi(t)), \mathrm{G}_{A y, A q, A s}^{*}(\varphi(t)), \mathrm{G}_{A \zeta, A \eta, A \theta}^{*}(\varphi(t)),\right. \\
& \mathrm{G}_{A x, A p, A p}^{*}(\varphi(t)), \mathrm{G}_{A y, A q, A q}^{*}(\varphi(t)), \mathrm{G}_{A \zeta, A \eta, A \eta}^{*}(\varphi(\mathrm{t})), \\
& \mathrm{G}_{A p, A r, A r}^{*}(\varphi(t)), \mathrm{G}_{A \mathrm{q}, A s, A s}^{*}(\varphi(t)), \mathrm{G}_{A \eta, A \theta, A \theta}^{*}(\varphi(t)), \\
& \left.\mathrm{G}_{A x, A r, A r}^{*}(\varphi(t)), \mathrm{G}_{A y, A s, A s}^{*}(\varphi(t)), \mathrm{G}_{A \zeta, A \theta, A \theta}^{*}(\varphi(t))\right\}
\end{aligned}
$$

for all $x, y, p, q, r, s, \zeta, \eta, \theta \in X$, where $T(X \times X \times X) \subset A(X), A$ is continuous and commutative with $T$. Then there exists a unique $\mathrm{u} \in \mathrm{X}$ such that $\mathrm{u}=\mathrm{Au}=\mathrm{T}(\mathrm{u}, \mathrm{u}, \mathrm{u})$.

Letting $A=I$ ( $\mathrm{I}$ is the identity mapping) in Theorem 3.28, we can obtain the following corollary.

Corollary 3.29. Let $\left(\mathrm{X}, \mathrm{G}^{*}, \Delta\right)$ be a complete PGM-space such that $\Delta$ is a $\mathrm{t}$-norm of $\mathrm{H}$-type. Let $\varphi: \mathbb{R}^{+} \rightarrow \mathbb{R}^{+}$be a gauge function such that $\varphi^{-1}(\{0\})=\{0\}, \varphi(t)>t$ and $\lim _{n \rightarrow \infty} \varphi^{n}(t)=+\infty$ for any $\mathrm{t}>0$. Let $\mathrm{T}: \mathrm{X} \times \mathrm{X} \times \mathrm{X} \rightarrow$ $\mathrm{X}$ be a mapping such that

$$
\begin{aligned}
& \mathrm{G}_{\mathrm{T}(x, y, \zeta), T(p, q, \eta), T(r, s, \theta)}^{*}(t) \geqslant \min \left\{\mathrm{G}_{x, p, r}^{*}(\varphi(t)), \mathrm{G}_{y, q, s}^{*}(\varphi(t)), \mathrm{G}_{\zeta, \eta, \theta}^{*}(\varphi(t)), \mathrm{G}_{x, p, p}^{*}(\varphi(t)),\right. \\
& \mathrm{G}_{\mathrm{y}, \mathrm{q}, \mathrm{q}}^{*}(\varphi(\mathrm{t})), \mathrm{G}_{\zeta, \eta, \eta}^{*}(\varphi(\mathrm{t})), \mathrm{G}_{\mathrm{p}, \mathrm{r}, \mathrm{r}}^{*}(\varphi(\mathrm{t})), \mathrm{G}_{\mathrm{q}, \mathrm{s}, \mathrm{s}}^{*}(\varphi(\mathrm{t})), \\
& \left.\mathrm{G}_{\eta, \theta, \theta}^{*}(\varphi(t)), \mathrm{G}_{x, r, r}^{*}(\varphi(t)), \mathrm{G}_{\mathrm{y}, \mathrm{s}, \mathrm{s}}^{*}(\varphi(\mathrm{t})), \mathrm{G}_{\zeta, \theta, \theta}^{*}(\varphi(t))\right\}
\end{aligned}
$$

for all $x, y, p, q, r, s, \zeta, \eta, \theta \in X$ and $t>0$. Then $T$ has a unique fixed point in $X$.

In a similar way, we can prove the following result.

Theorem 3.30. Let $\left(\mathrm{X}, \mathrm{G}^{*}, \Delta\right)$ be a complete PGM-space such that $\Delta$ is a $\mathrm{t}$-norm of $\mathrm{H}$-type. Let $\varphi: \mathbb{R}^{+} \rightarrow \mathbb{R}^{+}$be a gauge function such that $\varphi^{-1}(\{0\})=\{0\}, \varphi(t)<\mathrm{t}$ and $\lim _{\mathrm{n} \rightarrow \infty} \varphi^{\mathrm{n}}(\mathrm{t})=0$ for any $\mathrm{t}>0$. Let $\mathrm{T}: \mathrm{X} \times \mathrm{X} \times \mathrm{X} \rightarrow \mathrm{X}$ and $\mathrm{A}: \mathrm{X} \rightarrow \mathrm{X}$ be two mappings such that

$$
\begin{aligned}
& \mathrm{G}_{\mathrm{T}(x, y, \zeta), T(p, q, \eta), T(r, s, \theta)}^{*}(\varphi(t)) \geqslant \min \left\{\mathrm{G}_{A x, A p, A r}^{*}(t), G_{A y, A q, A s}^{*}(t), G_{A \zeta, A \eta, A \theta}^{*}(t), G_{A x, A p, A p}^{*}(t),\right. \\
& \mathrm{G}_{A y, A q, A q}^{*}(\mathrm{t}), \mathrm{G}_{A \zeta, A \eta, A \eta}^{*}(\mathrm{t}), \mathrm{G}_{A \mathrm{p}, A r, A r}^{*}(\mathrm{t}), \mathrm{G}_{A \mathrm{q}, A s, A s}^{*}(\mathrm{t}), \\
& \left.\mathrm{G}_{A \eta, A \theta, A \theta}^{*}(t), G_{A x, A r, A r}^{*}(t), G_{A y, A s, A s}^{*}(t), G_{A \zeta, A \theta, A \theta}^{*}(t)\right\}
\end{aligned}
$$

for all $x, y, p, q, r, s, \zeta, \eta, \theta \in X$, where $T(X \times X \times X) \subset A(X), A$ is continuous and commutative with $T$. Then there exists a unique $\mathrm{u} \in \mathrm{X}$ such that $\mathrm{u}=\mathrm{Au}=\mathrm{T}(\mathrm{u}, \mathrm{u}, \mathrm{u})$.

In Theorem 3.30, if we take $A=I$ ( $\mathrm{I}$ is the identity mapping), then we have the following corollary.

Corollary 3.31. Let $\left(\mathrm{X}, \mathrm{G}^{*}, \Delta\right)$ be a complete PGM-space such that $\Delta$ is a $\mathrm{t}$-norm of $\mathrm{H}$-type. Let $\varphi: \mathbb{R}^{+} \rightarrow \mathbb{R}^{+}$be a gauge function such that $\varphi^{-1}(\{0\})=\{0\}, \varphi(t)<\mathrm{t}$ and $\lim _{\mathrm{n} \rightarrow \infty} \varphi^{\mathrm{n}}(\mathrm{t})=0$ for any $\mathrm{t}>0$. Let $\mathrm{T}: \mathrm{X} \times \mathrm{X} \times \mathrm{X} \rightarrow \mathrm{X}$ be a mapping satisfying

$$
\begin{array}{r}
G_{T(x, y, \zeta), T(p, q, \eta), T(r, s, \theta)}^{*}(\varphi(t)) \geqslant \min \left\{G_{x, p, r}^{*}(t), G_{y, q, s}^{*}(t), G_{\zeta, \eta, \theta}^{*}(t), G_{x, p, p}^{*}(t), G_{y, q, q}^{*}(t), G_{\zeta, \eta, \eta}^{*}(t),\right. \\
\left.G_{p, r, r}^{*}(t), G_{q, s, s}^{*}(t), G_{\eta, \theta, \theta}^{*}(t), G_{x, r, r}^{*}(t), G_{y, s, s}^{*}(t), G_{\zeta, \theta, \theta}^{*}(t)\right\}
\end{array}
$$

for all $x, y, p, q, r, s, \zeta, \eta, \theta \in X$ and $t>0$. Then $T$ has a unique fixed point in $X$.

In Theorem 3.30, if we take $\varphi(t)=\lambda t(0<\lambda<1)$, then we have the following corollary. 
Corollary 3.32. Let $\left(\mathrm{X}, \mathrm{G}^{*}, \Delta\right)$ be a complete PGM-space such that $\Delta$ is a $\mathrm{t}$-norm of $\mathrm{H}$-type. Let $\mathrm{T}: \mathrm{X} \times \mathrm{X} \times \mathrm{X} \rightarrow \mathrm{X}$ and $\mathrm{A}: \mathrm{X} \rightarrow \mathrm{X}$ be two mappings such that

$$
\begin{aligned}
G_{T(x, y, \zeta), T(p, q, \eta), T(r, s, \theta)}^{*}(\lambda t) \geqslant \min & \left\{G_{A x, A p, A r}^{*}(t), G_{A y, A q, A s}^{*}(t), G_{A \zeta, A \eta, A \theta}^{*}(t), G_{A x, A p, A p}^{*}(t),\right. \\
& G_{A y, A q, A q}^{*}(t), G_{A \zeta, A \eta, A \eta}^{*}(t), G_{A p, A r, A r}^{*}(t), G_{A q, A s, A s}^{*}(t), \\
& \left.G_{A \eta, A \theta, A \theta}^{*}(t), G_{A x, A r, A r}^{*}(t), G_{A y, A s, A s}^{*}(t), G_{A \zeta, A \theta, A \theta}^{*}(t)\right\}
\end{aligned}
$$

for all $x, y, p, q, r, s, \zeta, \eta, \theta \in X$, where $\lambda \in(0,1), T(X \times X \times X) \subset A(X), A$ is continuous and commutative with $\mathrm{T}$. Then there exists a unique $\mathrm{u} \in \mathrm{X}$ such that $\mathrm{u}=\mathrm{Au}=\mathrm{T}(\mathrm{u}, \mathrm{u}, \mathrm{u})$.

Finally, by the same methods as in Theorems 3.23, 3.26, 3.28, and 3.30, we can obtain the following common quadruple fixed point results under probabilistic $\varphi$-contractive conditions in PGM-space.

Theorem 3.33. Let $\left(\mathrm{X}, \mathrm{G}^{*}, \Delta\right)$ be a complete PGM-space such that $\Delta$ is a $\mathrm{t}$-norm of $\mathrm{H}$-type. Let $\varphi: \mathbb{R}^{+} \rightarrow \mathbb{R}^{+}$be a gauge function such that $\varphi^{-1}(\{0\})=\{0\}, \varphi(t)<\mathrm{t}$ and $\lim _{\mathrm{n} \rightarrow \infty} \varphi^{\mathrm{n}}(\mathrm{t})=0$ for any $\mathrm{t}>0$. Let $\mathrm{T}: \mathrm{X} \times \mathrm{X} \times \mathrm{X} \times \mathrm{X} \rightarrow$ $\mathrm{X}$ and $\mathrm{A}: \mathrm{X} \rightarrow \mathrm{X}$ be two mappings such that

$$
\begin{aligned}
\mathrm{G}_{\mathrm{T}(x, y, \zeta, \sigma), T(p, q, \eta, \sigma), T(r, s, \theta, \tau)}^{*}(\varphi(t)) \geqslant & {\left[\mathrm{G}_{A x, A p, A r}^{*}(t) G_{A y, A q, A s}^{*}(t) G_{A \zeta, A \eta, A \theta}^{*}(t) G_{A \sigma, A \sigma, A \tau}^{*}(t)\right.} \\
& \times G_{A x, A p, A p}^{*}(t) G_{A y, A q, A q}^{*}(t) G_{A \zeta, A \eta, A \eta}^{*}(t) G_{A \sigma, A \sigma, A \sigma}^{*}(t) \\
& \times G_{A p, A r, A r}^{*}(t) G_{A q, A s, A s}^{*}(t) G_{A \eta, A \theta, A \theta}^{*}(t) G_{A \sigma, A \tau, A \tau}^{*}(t) \\
& \left.\times G_{A x, A r, A r}^{*}(t) G_{A y, A s, A s}^{*}(t) G_{A \zeta, A \theta, A \theta}^{*}(t) G_{A \sigma, A \tau, A \tau}^{*}(t)\right]^{\frac{1}{16}}
\end{aligned}
$$

for all $x, y, p, q, r, s, \zeta, \eta, \theta, \sigma, \sigma, \tau \in X$, where $T(X \times X \times X \times X) \subset A(X), A$ is continuous and commutative with $\mathrm{T}$. Then there exists a unique $\mathrm{u} \in \mathrm{X}$ such that $\mathrm{u}=\mathrm{Au}=\mathrm{T}(\mathrm{u}, \mathrm{u}, \mathrm{u}, \mathrm{u})$.

In Theorem 3.33, if we take $A=I$ ( $\mathrm{I}$ is the identity mapping), then we have the following corollary.

Corollary 3.34. Let $\left(\mathrm{X}, \mathrm{G}^{*}, \Delta\right)$ be a complete PGM-space such that $\Delta$ is a $\mathrm{t}$-norm of $\mathrm{H}$-type. Let $\varphi: \mathbb{R}^{+} \rightarrow \mathbb{R}^{+}$be a gauge function such that $\varphi^{-1}(\{0\})=\{0\}, \varphi(t)<\mathrm{t}$ and $\lim _{n \rightarrow \infty} \varphi^{\mathrm{n}}(\mathrm{t})=0$ for any $\mathrm{t}>0$. Let $\mathrm{T}: \mathrm{X} \times \mathrm{X} \times \mathrm{X} \times \mathrm{X} \rightarrow$ $\mathrm{X}$ be a mapping satisfying

$$
\begin{aligned}
& \mathrm{G}_{\mathrm{T}(\mathrm{x}, \mathrm{y}, \zeta, \sigma), T(p, q, \eta, \sigma), T(r, s, \theta, \tau)}^{*}(\varphi(t)) \geqslant\left[\mathrm{G}_{x, p, r}^{*}(t) G_{y, q, s}^{*}(t) G_{\zeta, \eta, \theta}^{*}(t) G_{\sigma, \sigma, \tau}^{*}(t) G_{x, p, p}^{*}(t) G_{y, q, q}^{*}(t)\right. \\
& \times G_{\zeta, \eta, \eta}^{*}(t) G_{\sigma, \sigma, \sigma}^{*}(t) G_{p, r, r}^{*}(t) G_{q, s, s}^{*}(t) G_{\eta, \theta, \theta}^{*}(t) G_{\sigma, \tau, \tau}^{*}(t) \\
& \left.\times G_{x, r, r}^{*}(t) G_{y, s, s}^{*}(t) G_{\zeta, \theta, \theta}^{*}(t) G_{\sigma, \tau, \tau}^{*}(t)\right]^{\frac{1}{16}}
\end{aligned}
$$

for all $x, y, p, q, r, s, \zeta, \eta, \theta, \sigma, \sigma, \tau \in X$ and $t>0$. Then $T$ has a unique fixed point in $X$.

In Theorem 3.33, if we take $\varphi(t)=\lambda t(\lambda \in(0,1))$, then we have the following corollary.

Corollary 3.35. Let $\left(\mathrm{X}, \mathrm{G}^{*}, \Delta\right)$ be a complete PGM-space such that $\Delta$ is a $\mathrm{t}$-norm of $\mathrm{H}$-type. Let $\mathrm{T}: \mathrm{X} \times \mathrm{X} \times \mathrm{X} \times$ $\mathrm{X} \rightarrow \mathrm{X}$ and $\mathrm{A}: \mathrm{X} \rightarrow \mathrm{X}$ be two mappings such that

$$
\begin{aligned}
& \mathrm{G}_{\mathrm{T}(x, y, \zeta, \sigma), T(p, q, \eta, \sigma), T(r, s, \theta, \tau)}^{*}(\lambda t) \geqslant\left[\mathrm{G}_{A x, A p, A r}^{*}(t) G_{A y, A q, A s}^{*}(t) G_{A \zeta, A \eta, A \theta}^{*}(t) G_{A \sigma, A \sigma, A \tau}^{*}(t)\right. \\
& \times G_{A x, A p, A p}^{*}(t) G_{A y, A q, A q}^{*}(t) G_{A \zeta, A \eta, A \eta}^{*}(t) G_{A \sigma, A \sigma, A \sigma}^{*}(t) \\
& \times G_{A p, A r, A r}^{*}(t) G_{A q, A s, A s}^{*}(t) G_{A \eta, A \theta, A \theta}^{*}(t) G_{A \sigma, A \tau, A \tau}^{*}(t) \\
& \left.\times G_{A x, A r, A r}^{*}(t) G_{A y, A s, A s}^{*}(t) G_{A \zeta, A \theta, A \theta}^{*}(t) G_{A \sigma, A \tau, A \tau}^{*}(t)\right]^{\frac{1}{16}}
\end{aligned}
$$

for all $x, y, p, q, r, s, \zeta, \eta, \theta, \sigma, \sigma, \tau \in X$, where $\lambda \in(0,1), T(X \times X \times X \times X) \subset A(X), A$ is continuous and commutative with $\mathrm{T}$. Then there exists a unique $\mathrm{u} \in \mathrm{X}$ such that $\mathrm{u}=\mathrm{Au}=\mathrm{T}(\mathrm{u}, \mathrm{u}, \mathrm{u}, \mathrm{u})$. 
Theorem 3.36. Let $\left(\mathrm{X}, \mathrm{G}^{*}, \Delta\right)$ be a complete PGM-space such that $\Delta$ is a $\mathrm{t}$-norm of $\mathrm{H}$-type. Let $\varphi: \mathbb{R}^{+} \rightarrow \mathbb{R}^{+}$ be a gauge function such that $\varphi^{-1}(\{0\})=\{0\}, \varphi(t)>\mathrm{t}$ and $\lim _{\mathrm{n} \rightarrow \infty} \varphi^{\mathrm{n}}(\mathrm{t})=+\infty$ for any $\mathrm{t}>0$. Let $\mathrm{T}$ : $\mathrm{X} \times \mathrm{X} \times \mathrm{X} \times \mathrm{X} \rightarrow \mathrm{X}$ and $\mathrm{A}: \mathrm{X} \rightarrow \mathrm{X}$ be two mappings such that

$$
\begin{aligned}
\mathrm{G}_{\mathrm{T}(\mathrm{x}, \mathrm{y}, \zeta, \sigma), T(p, q, \eta, \sigma), T(\mathrm{r}, \mathrm{s}, \theta, \tau)}^{*}(t) \geqslant & {\left[G_{A x, A p, A r}^{*}(\varphi(t)) G_{A y, A q, A s}^{*}(\varphi(t)) G_{A \zeta, A \eta, A \theta}^{*}(\varphi(t)) G_{A \sigma, A \sigma, A \tau}^{*}(\varphi(t))\right.} \\
& \times G_{A x, A p, A p}^{*}(\varphi(t)) G_{A y, A q, A q}^{*}(\varphi(t)) G_{A \zeta, A \eta, A \eta}^{*}(\varphi(t)) G_{A \sigma, A \sigma, A \sigma}^{*}(\varphi(t)) \\
& \times G_{A p, A r, A r}^{*}(\varphi(t)) G_{A q, A s, A s}^{*}(\varphi(t)) G_{A \eta, A \theta, A \theta}^{*}(\varphi(t)) G_{A \sigma, A \tau, A \tau}^{*}(\varphi(t)) \\
& \left.\times G_{A x, A r, A r}^{*}(\varphi(t)) G_{A y, A s, A s}^{*}(\varphi(t)) G_{A \zeta, A \theta, A \theta}^{*}(\varphi(t)) G_{A \sigma, A \tau, A \tau}^{*}(\varphi(t))\right]^{\frac{1}{16}}
\end{aligned}
$$

for all $x, y, p, q, r, s, \zeta, \eta, \theta, \sigma, \sigma, \tau \in X$, where $T(X \times X \times X \times X) \subset A(X), A$ is continuous and commutative with $\mathrm{T}$. Then there exists a unique $\mathrm{u} \in \mathrm{X}$ such that $\mathrm{u}=\mathrm{Au}=\mathrm{T}(\mathrm{u}, \mathrm{u}, \mathrm{u}, \mathrm{u})$.

Letting $A=I$ (I is the identity mapping) in Theorem 3.36, we can obtain the following corollary.

Corollary 3.37. Let $\left(\mathrm{X}, \mathrm{G}^{*}, \Delta\right)$ be a complete PGM-space such that $\Delta$ is a $\mathrm{t}$-norm of $\mathrm{H}$-type. Let $\varphi: \mathbb{R}^{+} \rightarrow \mathbb{R}^{+}$ be a gauge function such that $\varphi^{-1}(\{0\})=\{0\}, \varphi(t)>\mathrm{t}$ and $\lim _{\mathrm{n} \rightarrow \infty} \varphi^{\mathrm{n}}(\mathrm{t})=+\infty$ for any $\mathrm{t}>0$. Let $\mathrm{T}$ : $\mathrm{X} \times \mathrm{X} \times \mathrm{X} \times \mathrm{X} \rightarrow \mathrm{X}$ be a mapping satisfying

$$
\begin{aligned}
& \mathrm{G}^{*} \mathrm{~T}(\mathrm{x}, \mathrm{y}, \zeta, \sigma), T(p, q, \eta, \sigma), T(r, s, \theta, \tau)(t) \\
& \geqslant\left[G_{x, p, r}^{*}(\varphi(t)) G_{y, q, s}^{*}(\varphi(t)) G_{\zeta, \eta, \theta}^{*}(\varphi(t)) G_{\sigma, \sigma, \tau}^{*}(\varphi(t)) G_{x, p, p}^{*}(\varphi(t)) G_{y, q, q}^{*}(\varphi(t))\right. \\
& \times G_{\zeta, \eta, \eta}^{*}(\varphi(t)) G_{\sigma, \sigma, \sigma}^{*}(\varphi(t)) G_{p, r, r}^{*}(\varphi(t)) G_{q, s, s}^{*}(\varphi(t)) G_{\eta, \theta, \theta}^{*}(\varphi(t)) G_{\sigma, \tau, \tau}^{*}(\varphi(t)) \\
& \left.\times G_{x, r, r}^{*}(\varphi(t)) G_{y, s, s}^{*}(\varphi(t)) G_{\zeta, \theta, \theta}^{*}(\varphi(t)) G_{\sigma, \tau, \tau}^{*}(\varphi(t))\right]^{\frac{1}{16}}
\end{aligned}
$$

for all $x, y, p, q, r, s, \zeta, \eta, \theta, \sigma, \sigma, \tau \in X$ and $t>0$. Then $T$ has a unique fixed point in $X$.

Theorem 3.38. Let $\left(\mathrm{X}, \mathrm{G}^{*}, \Delta\right)$ be a complete PGM-space such that $\Delta$ is a $\mathrm{t}$-norm of $\mathrm{H}$-type. Let $\varphi: \mathbb{R}^{+} \rightarrow \mathbb{R}^{+}$ be a gauge function such that $\varphi^{-1}(\{0\})=\{0\}, \varphi(\mathrm{t})>\mathrm{t}$ and $\lim _{\mathrm{n} \rightarrow \infty} \varphi^{\mathrm{n}}(\mathrm{t})=+\infty$ for any $\mathrm{t}>0$. Let $\mathrm{T}$ : $\mathrm{X} \times \mathrm{X} \times \mathrm{X} \times \mathrm{X} \rightarrow \mathrm{X}$ and $\mathrm{A}: \mathrm{X} \rightarrow \mathrm{X}$ be two mappings such that

$$
\begin{aligned}
G^{*} T(x, y, \zeta, \sigma), T(p, q, \eta, \sigma), T(r, s, \theta, \tau) & (t) \\
\geqslant & \min \left\{G_{A x, A p, A r}^{*}(\varphi(t)), G_{A y, A q, A s}^{*}(\varphi(t)), G_{A \zeta, A \eta, A \theta}^{*}(\varphi(t)), G_{A \sigma, A \sigma, A \tau}^{*}(\varphi(t)),\right. \\
& G_{A x, A p, A p}^{*}(\varphi(t)), G_{A y, A q, A q}^{*}(\varphi(t)), G_{A \zeta, A \eta, A \eta}^{*}(\varphi(t)), G_{A \sigma, A \sigma, A \sigma}^{*}(\varphi(t)), \\
& G_{A p, A r, A r}^{*}(\varphi(t)), G_{A q, A s, A s}^{*}(\varphi(t)), G_{A \eta, A \theta, A \theta}^{*}(\varphi(t)), G_{A \sigma, A \tau, A \tau}^{*}(\varphi(t)), \\
& \left.G_{A x, A r, A r}^{*}(\varphi(t)), G_{A y, A s, A s}^{*}(\varphi(t)), G_{A \zeta, A \theta, A \theta}^{*}(\varphi(t)), G_{A \sigma, A \tau, A \tau}^{*}(\varphi(t))\right\}
\end{aligned}
$$

for all $x, y, p, q, r, s, \zeta, \eta, \theta, \sigma, \sigma, \tau \in X$, where $T(X \times X \times X \times X) \subset A(X), A$ is continuous and commutative with $T$. Then there exists a unique $\mathrm{u} \in \mathrm{X}$ such that $\mathrm{u}=\mathrm{Au}=\mathrm{T}(\mathrm{u}, \mathrm{u}, \mathrm{u}, \mathrm{u})$.

Letting $A=I$ (I is the identity mapping) in Theorem 3.38, we can obtain the following corollary.

Corollary 3.39. Let $\left(\mathrm{X}, \mathrm{G}^{*}, \Delta\right)$ be a complete PGM-space such that $\Delta$ is a $\mathrm{t}$-norm of $\mathrm{H}$-type. Let $\varphi: \mathbb{R}^{+} \rightarrow \mathbb{R}^{+}$ be a gauge function such that $\varphi^{-1}(\{0\})=\{0\}, \varphi(t)>\mathrm{t}$ and $\lim _{\mathrm{n} \rightarrow \infty} \varphi^{\mathrm{n}}(\mathrm{t})=+\infty$ for any $\mathrm{t}>0$. Let $\mathrm{T}$ : $\mathrm{X} \times \mathrm{X} \times \mathrm{X} \times \mathrm{X} \rightarrow \mathrm{X}$ be a mapping such that

$$
\begin{array}{r}
G_{T(x, y, \zeta, \sigma), T(p, q, \eta, \sigma), T(r, s, \theta, \tau)}^{*}(t) \geqslant \min \left\{G_{x, p, r}^{*}(\varphi(t)), G_{y, q, s}^{*}(\varphi(t)), G_{\zeta, \eta, \theta}^{*}(\varphi(t)), G_{\sigma, \sigma, \tau}^{*}(\varphi(t)),\right. \\
G_{x, p, p}^{*}(\varphi(t)), G_{y, q, q}^{*}(\varphi(t)), G_{\zeta, \eta, \eta}^{*}(\varphi(t)), G_{\sigma, \sigma, \sigma}^{*}(\varphi(t)), \\
G_{p, r, r}^{*}(\varphi(t)), G_{q, s, s}^{*}(\varphi(t)), G_{\eta, \theta, \theta}^{*}(\varphi(t)), G_{\sigma, \tau, \tau}^{*}(\varphi(t)), \\
\\
\left.G_{x, r, r}^{*}(\varphi(t)), G_{y, s, s}^{*}(\varphi(t)), G_{\zeta, \theta, \theta}^{*}(\varphi(t)), G_{\sigma, \tau, \tau}^{*}(\varphi(t))\right\}
\end{array}
$$

for all $x, y, p, q, r, s, \zeta, \eta, \theta, \sigma, \sigma, \tau \in X$ and $t>0$. Then $T$ has a unique fixed point in $X$. 
Theorem 3.40. Let $\left(\mathrm{X}, \mathrm{G}^{*}, \Delta\right)$ be a complete PGM-space such that $\Delta$ is a $\mathrm{t}$-norm of $\mathrm{H}$-type. Let $\varphi: \mathbb{R}^{+} \rightarrow \mathbb{R}^{+}$be a gauge function such that $\varphi^{-1}(\{0\})=\{0\}, \varphi(t)<\mathrm{t}$ and $\lim _{\mathrm{n} \rightarrow \infty} \varphi^{\mathrm{n}}(\mathrm{t})=0$ for any $\mathrm{t}>0$. Let $\mathrm{T}: \mathrm{X} \times \mathrm{X} \times \mathrm{X} \times \mathrm{X} \rightarrow$ $\mathrm{X}$ and $\mathrm{A}: \mathrm{X} \rightarrow \mathrm{X}$ be two mappings such that

$$
\begin{aligned}
& G_{T(x, y, \zeta, \sigma), T(p, q, \eta, \sigma), T(r, s, \theta, \tau)}^{*}(\varphi(t)) \geqslant \min \left\{G_{A x, A p, A r}^{*}(t), G_{A y, A q, A s}^{*}(t), G_{A \zeta, A \eta, A \theta}^{*}(t), G_{A \sigma, A \sigma, A \tau}^{*}(t),\right. \\
& \mathrm{G}_{A x, A p, A p}^{*}(\mathrm{t}), \mathrm{G}_{A y, A q, A q}^{*}(\mathrm{t}), \mathrm{G}_{A \zeta, A \eta, A \eta}^{*}(\mathrm{t}), \mathrm{G}_{A \sigma, A \sigma, A \sigma}^{*}(\mathrm{t}), \\
& G_{A p, A r, A r}^{*}(t), G_{A q, A s, A s}^{*}(t), G_{A \eta, A \theta, A \theta}^{*}(t), G_{A \sigma, A \tau, A \tau}^{*}(t), \\
& \left.\mathrm{G}_{A x, A r, A r}^{*}(t), G_{A y, A s, A s}^{*}(t), G_{A \zeta, A \theta, A \theta}^{*}(t), G_{A \sigma, A \tau, A \tau}^{*}(t)\right\}
\end{aligned}
$$

for all $x, y, p, q, r, s, \zeta, \eta, \theta, \sigma, \sigma, \tau \in X$, where $T(X \times X \times X \times X) \subset A(X), A$ is continuous and commutative with $T$. Then there exists a unique $\mathrm{u} \in \mathrm{X}$ such that $\mathrm{u}=\mathrm{Au}=\mathrm{T}(\mathrm{u}, \mathrm{u}, \mathrm{u}, \mathrm{u})$.

In Theorem 3.40, if we take $A=I$ ( $\mathrm{I}$ is the identity mapping), then we have the following corollary.

Corollary 3.41. Let $\left(\mathrm{X}, \mathrm{G}^{*}, \Delta\right)$ be a complete PGM-space such that $\Delta$ is a $\mathrm{t}$-norm of $\mathrm{H}$-type. Let $\varphi: \mathbb{R}^{+} \rightarrow \mathbb{R}^{+}$be a gauge function such that $\varphi^{-1}(\{0\})=\{0\}, \varphi(t)<\mathrm{t}$ and $\lim _{n \rightarrow \infty} \varphi^{\mathrm{n}}(\mathrm{t})=0$ for any $\mathrm{t}>0$. Let $\mathrm{T}: \mathrm{X} \times \mathrm{X} \times \mathrm{X} \times \mathrm{X} \rightarrow$ $\mathrm{X}$ be a mapping satisfying

$$
\begin{aligned}
& \mathrm{G}_{\mathrm{T}(x, y, \zeta, \sigma), T(p, q, \eta, \sigma), T(r, s, \theta, \tau)}^{*}(\varphi(t)) \geqslant \min \left\{\mathrm{G}_{x, p, r}^{*}(t), \mathrm{G}_{y, q, s}^{*}(t), \mathrm{G}_{\zeta, \eta, \theta}^{*}(t), \mathrm{G}_{\sigma, \sigma, \tau}^{*}(t),\right. \\
& G_{x, p, p}^{*}(t), G_{y, q, q}^{*}(t), G_{\zeta, \eta, \eta}^{*}(t), G_{\sigma, \sigma, \sigma}^{*}(t), \\
& G_{p, r, r}^{*}(t), G_{q, s, s}^{*}(t), G_{\eta, \theta, \theta}^{*}(t), G_{\sigma, \tau, \tau}^{*}(t), \\
& \left.\mathrm{G}_{x, r, r}^{*}(t), G_{y, s, s}^{*}(t), G_{\zeta, \theta, \theta}^{*}(t), G_{\sigma, \tau, \tau}^{*}(t)\right\}
\end{aligned}
$$

for all $x, y, p, q, r, s, \zeta, \eta, \theta, \sigma, \sigma, \tau \in X$ and $t>0$. Then $T$ has a unique fixed point in $X$.

In Theorem 3.40, if we take $\varphi(t)=\lambda t(0<\lambda<1)$, then we have the following corollary.

Corollary 3.42. Let $\left(\mathrm{X}, \mathrm{G}^{*}, \Delta\right)$ be a complete PGM-space such that $\Delta$ is a t-norm of $\mathrm{H}$-type. Let $\mathrm{T}: \mathrm{X} \times \mathrm{X} \times \mathrm{X} \times$ $\mathrm{X} \rightarrow \mathrm{X}$ and $\mathrm{A}: \mathrm{X} \rightarrow \mathrm{X}$ be two mappings such that

$$
\begin{aligned}
& G_{T(x, y, \zeta, \sigma), T(p, q, \eta, \sigma), T(r, s, \theta, \tau)}^{*}(\lambda t) \geqslant \min \left\{G_{A x, A p, A r}^{*}(t), G_{A y, A q, A s}^{*}(t), G_{A \zeta, A \eta, A \theta}^{*}(t) G_{A \sigma, A \sigma, A \tau}^{*}(t),\right. \\
& \mathrm{G}_{A x, A p, A p}^{*}(\mathrm{t}), \mathrm{G}_{A y, A q, A q}^{*}(\mathrm{t}), \mathrm{G}_{A \zeta, A \eta, A \eta}^{*}(\mathrm{t}), \mathrm{G}_{A \sigma, A \sigma, A \sigma}^{*}(\mathrm{t}), \\
& \mathrm{G}_{A p, A r, A r}^{*}(t), G_{A q, A s, A s}^{*}(t), G_{A \eta, A \theta, A \theta}^{*}(t), G_{A \sigma, A \tau, A \tau}^{*}(t), \\
& \left.\mathrm{G}_{A x, A r, A r}^{*}(t), G_{A y, A s, A s}^{*}(t), G_{A \zeta, A \theta, A \theta}^{*}(t), G_{A \sigma, A \tau, A \tau}^{*}(t)\right\}
\end{aligned}
$$

for all $x, y, p, q, r, s, \zeta, \eta, \theta, \sigma, \sigma, \tau \in X$, where $\lambda \in(0,1), T(X \times X \times X \times X) \subset A(X), A$ is continuous and commutative with $\mathrm{T}$. Then there exists a unique $\mathrm{u} \in \mathrm{X}$ such that $\mathrm{u}=\mathrm{Au}=\mathrm{T}(\mathrm{u}, \mathrm{u}, \mathrm{u}, \mathrm{u})$.

\section{An example}

In this section, we give the following example to illustrate Theorem 3.11.

Example 4.1. Let $X=[0, \infty), \Delta=\Delta_{\mathrm{p}}$. Then $\Delta$ is a t-norm of H-type. Define the mapping $\mathrm{G}^{*}: \mathrm{X}^{3} \times$ $[0, \infty) \rightarrow[0, \infty)$ by

$$
G_{x, y, z}^{*}(t)= \begin{cases}1, & t \leqslant 0, \\ e^{-\frac{|x-y|+|y-z|+|z-x|}{2 t}}, & t>0\end{cases}
$$

for all $x, y, z \in X$. Then $\left(X, G^{*}, \Delta_{p}\right)$ is a PGM-space. In fact, it is easy to check that $\mathrm{G}^{*}$ satisfies (PGM-1)(PGM-3). Next we show $G^{*}(x, y, z)(s+t) \geqslant \Delta\left(G^{*}(x, a, a)(s), G^{*}(a, y, y)(t)\right)=G^{*}(x, a, a)(s) G^{*}(a, y, y)(t)$ 
for all $x, y, z, a \in X$ and all $s, t>0$. In fact, since

$$
\begin{aligned}
\frac{|x-y|+|y-z|+|z-x|}{2(s+t)} & \leqslant \frac{|x-a|+|a-y|+|y-z|+|z-a|+|a-x|}{2(s+t)} \\
& =\frac{2|x-a|}{2(s+t)}+\frac{|a-y|+|y-z|+|z-a|}{2(s+t)} \\
& <\frac{2|x-a|}{2 s}+\frac{|a-y|+|y-z|+|z-a|}{2 t},
\end{aligned}
$$

we have

$$
\begin{aligned}
\mathrm{G}^{*}(x, y, z)(s+t) & =e^{-\frac{|x-y|+|y-z|+|z-x|}{2(s+t)}} \\
& \geqslant e^{-\left(\frac{2|x-a|}{2 s}+\frac{|a-y|+|y-z|+|z-a|}{2 t}\right)}=G_{x, a, a}^{*}(s) G_{a, y, z}^{*}(t) .
\end{aligned}
$$

This shows that $\mathrm{G}^{*}$ satisfies (PGM-4). Hence $\left(X, \mathrm{G}^{*}, \Delta_{\mathfrak{p}}\right)$ is a Menger PGM-space.

Suppose that $\varphi(t)=\frac{t}{2}$. Let $T(u, v)=u+v, A u=3 u$ for all $u, v \in X$ and $T(X, X) \subset A(X)$, and let $A$ be continuous and commutative with $T$. For each $x, y, p, q, h, l \in X$ and $t>0$, since

$$
\begin{aligned}
& \frac{|(x+y)-(p+q)|+|(p+q)-(h+l)|+|(h+l)-(x+y)|}{t} \\
&= \frac{2|(x+y)-(p+q)|+2|(p+q)-(h+l)|+2|(h+l)-(x+y)|}{2 t} \\
& \leqslant \frac{(|x-p|+|p-h|+|h-x|)+(|y-q|+|q-l|+|l-y|)}{2 t} \\
&+\frac{2|x-p|+2|y-q|+2|p-h|+2|q-l|+2|h-x|+2|l-y|}{2 t} \\
&=\left(\frac{3(|x-p|+|p-h|+|h-x|)}{t}+\frac{3(|y-q|+|q-l|+|l-y|)}{t}\right. \\
&\left.+\frac{6|x-p|}{t}+\frac{6|y-q|}{t}+\frac{6|p-h|}{t}+\frac{6|q-l|}{t}+\frac{6|h-x|}{t}+\frac{6|l-y|}{t}\right) \times \frac{1}{6} \\
&=\left(\frac{(3|x-p|+|p-h|+|h-x|)}{t}+\frac{3(|y-q|+|q-l|+|l-y|)}{t}\right. \\
&\left.+\frac{2|3 x-3 p|}{t}+\frac{2|3 y-3 q|}{t}+\frac{2|3 p-3 h|}{t}+\frac{2|3 q-3 l|}{t}+\frac{2|3 h-3 x|}{t}+\frac{2|3 l-3 y|}{t}\right) \times \frac{1}{6} \\
& \triangleq \Omega(x, y, p, q, h, l) \times \frac{1}{6},
\end{aligned}
$$

we get that

$$
\begin{aligned}
& \mathrm{G}_{\mathrm{T}(x, y), T(p, q), T(h, l)}^{*}\left(\frac{\mathrm{t}}{2}\right) \\
& =e^{-\frac{|(x+y)-(p+q)|+|(p+q)-(h+l)|+|(h+l)-(x+y)|}{t}} \\
& \geqslant e^{-\Omega(x, y, p, q, h, l) \times \frac{1}{6}} \\
& =\left[e^{-\frac{3(|x-p|+|p-h|+|h-x|)}{t}} \times e^{-\frac{3(|y-q|+|q-l|+|l-y|)}{t}} \times e^{-\frac{2|3 x-3 p|}{t}}\right.
\end{aligned}
$$




$$
\begin{aligned}
& \left.\times e^{-\frac{2|3 y-3 q|}{t}} \times e^{-\frac{2|3 p-3 h|}{t}} \times e^{-\frac{2|3 q-3 l|}{t}} \times e^{-\frac{2|3 h-3 x|}{t}} \times e^{-\frac{2|3 l-3 y|}{t}}\right]^{\frac{1}{6}} \\
= & {\left[G_{A x, A p, A h}^{*}(t) G_{A y, A q, A l}^{*}(t) G_{A x, A p, A p}^{*}(t) G_{A y, A q, A q}^{*}(t)\right.} \\
& \left.\left.\times G_{A p, A h, A h}^{*}(t) G_{A q, A l, A l}^{*}(t) G_{A x, A h, A h}^{*}(t) G_{A y, A l, A l}^{*}(t)\right)\right]^{\frac{1}{6}} .
\end{aligned}
$$

Thus all the conditions of Theorem 3.11 are satisfied. Therefore, 0 is the unique common coupled fixed point of $T$ and $A$.

\section{Acknowledgment}

The authors would like to express their sincere thanks to the anonymous referees for their great efforts to improve this paper.

This work was supported by the Application Basic Research Plan Key Basic Research Project of Hebei Province of China (Grant No. 16964213D), the Fundamental Research Funds for the Central Universities (Grant Nos. 2015ZD29 and 13ZD19), the Higher School Science Research Funds of Hebei Province of China (Grant No. Z2015137) and the Natural Science Foundation of Hebei University (Grant No. 799207217073).

\section{References}

[1] R. P. Agarwal, E. Karapınar, Remarks on some coupled fixed point theorems in G-metric spaces, Fixed Point Theory Appl., 2013 (2013), 33 pages. 3.3, 3.5

[2] V. Berinde, M. Borcut, Tripled fixed point theorems for contractive type mappings in partially ordered metric spaces, Nonlinear Anal., 74 (2011), 4889-4897. 1, 2

[3] L. Ćirić, R. P. Agarwal, B. Samet, Mixed monotone-generalized contractions in partially ordered probabilistic metric spaces, Fixed Point Theory Appl., 2011 (2011), 13 pages. 1

[4] J.-X. Fang, Common fixed point theorems of compatible and weakly compatible maps in Menger spaces, Nonlinear Anal., 71 (2009), 1833-1843. 1

[5] T. Gnana Bhaskar, V. Lakshmikantham, Fixed point theorems in partially ordered metric spaces and applications, Nonlinear Anal., 65 (2006), 1379-1393. 1, 1

[6] D. J. Guo, V. Lakshmikantham, Coupled fixed points of nonlinear operators with applications, Nonlinear Anal., 11 (1987), 623-632. 1

[7] O. Hadžić, A fixed point theorem in Menger spaces, Publ. Inst. Math. (Beograd) (N.S.), 20 (1979), 107-112. 2.2

[8] O. Hadžić, Fixed point theorems for multivalued mappings in probabilistic metric spaces, Fuzzy Sets and Systems, 88 (1997), 219-226. 2

[9] J. Jachymski, On probabilistic ф-contractions on Menger spaces, Nonlinear Anal., 73 (2010), 2199-2203. 2.10

[10] M. Jleli, E. Karapınar, B. Samet, Further generalizations of the Banach contraction principle, J. Inequal. Appl., 2014 (2014), 9 pages. 1

[11] M. Jleli, E. Karapınar, B. Samet, On cyclic $(\psi, \phi)$-contractions in Kaleva-Seikkala's type fuzzy metric spaces, J. Intell. Fuzzy Systems, 27 (2014), 2045-2053. 1

[12] E. Karapinar, N. V. Luong, Quadruple fixed point theorems for nonlinear contractions, Comput. Math. Appl., 64 (2012), 1839-1848. 1, 3

[13] V. Lakshmikantham, L. Ćirić, Coupled fixed point theorems for nonlinear contractions in partially ordered metric spaces, Nonlinear Anal., 70 (2009), 4341-4349. 1

[14] T. Luo, C.-X. Zhu, Z.-Q. Wu, Tripled common fixed point theorems under probabilistic $\phi$-contractive conditions in generalized Menger probabilistic metric spaces, Fixed Point Theory Appl., 2014 (2014), 17 pages. 1

[15] K. Menger, Statistical metrics, Proc. Nat. Acad. Sci. U. S. A., 28 (1942), 535-537. 2.4

[16] Z. Mustafa, B. Sims, A new approach to generalized metric spaces, J. Nonlinear Convex Anal., 7 (2006), 289-297. 2.5

[17] V. I. Opoĭcev, Heterogeneous and combined-concave operators, (Russian) Sibirsk. Mat. Ž., 16 (1975), 781-792. 1

[18] B. Samet, On the approximation of fixed points for a new class of generalized Berinde mappings, Carpathian J. Math., 32 (2016), 363-374. 1

[19] B. Schweizer, A. Sklar, Probabilistic metric spaces, North-Holland Series in Probability and Applied Mathematics, North-Holland Publishing Co., New York, (1983). 2.1

[20] S. Sedghi, I. Altun, N. Shobe, Coupled fixed point theorems for contractions in fuzzy metric spaces, Nonlinear Anal., 72 (2010), 1298-1304. 2.12 
[21] J. Wu, Some fixed-point theorems for mixed monotone operators in partially ordered probabilistic metric spaces, Fixed Point Theory Appl., 2014 (2014), 12 pages. 1

[22] J.-Z. Xiao, X.-H. Zhu, Y.-F. Cao, Common coupled fixed point results for probabilistic $\phi$-contractions in Menger spaces, Nonlinear Anal., 74 (2011), 4589-4600. 1, 3.12, 3.18

[23] C.-L. Zhou, S.-H. Wang, L. Ćirić, S. M. Alsulami, Generalized probabilistic metric spaces and fixed point theorems, Fixed Point Theory Appl., 2014 (2014), 15 pages. 1, 2, 2.6, 2.7, 2.8, 2.9 